Modern Physics Letters A

(C) World Scientific Publishing Company

IPHC-PHENO-12-01

\title{
BEYOND THE MINIMAL SUPERSYMMETRIC STANDARD MODEL: FROM THEORY TO PHENOMENOLOGY
}

\author{
BENJAMIN FUKS \\ Institut Pluridisciplinaire Hubert Curien/Département Recherches Subatomiques, Université de \\ Strasbourg/CNRS-IN2P3, 23 rue du Loess, F-67037 Strasbourg, France \\ benjamin.fuks@iphc.cnrs.fr
}

\begin{abstract}
Thanks to the latest development in the field of Monte Carlo event generators and satellite programs allowing for a straightforward implementation of any beyond the Standard Model theory in those tools, studying the property of any softly-broken supersymmetric theory is become an easy task. We illustrate this statement in the context of two non-minimal supersymmetric theories, namely the Minimal Supersymmetric Standard Model with $R$-parity violation and the Minimal $R$-symmetric Supersymmetric Standard Model and choose to probe interaction vertices involving a non-standard color structure and the sector of the top quark. We show how to efficiently implement these theories in the Mathematica package FeynRules and use its interfaces to Monte Carlo tools for phenomenological studies. For the latter, we employ the latest version of the MADGRAPH program.
\end{abstract}

Keywords: Supersymmetry; Monte Carlo event generators; hadron colliders.

11.30.Pb; 12.60.Jv; $13.85 . \mathrm{Hd} ; 13.85 .-\mathrm{t} ; 14.80 . \mathrm{Ly}$.

\section{Introduction}

Many extensions to the Standard Model of particle physics have been proposed over the last forty years. In particular, weak scale supersymmetry, and especially its minimal version dubbed the Minimal Supersymmetric Standard Model (MSSM) 112 , is one of the most theoretically and experimentally studied alternatives of the Standard Model. Supersymmetry is a symmetry relating particles with opposite statistics. In the MSSM, one single fermionic (bosonic) superpartner is assigned to each of the Standard Model bosonic (fermionic) degree of freedom, with the exception of the Higgs sector which contains two doublets of scalar Higgs fields, together with their fermionic superpartners. In this way, several conceptual problems of the Standard Model are addressed, such as the large hierarchy between the Planck and the electroweak scale, gauge coupling unification at high energy or the existence of a candidate for dark matter. However, since the superpartners of the Standard Model particles have not yet been observed, supersymmetry has to be broken at low energies. In addition, in order to remain a viable solution to the hierarchy problem, this breaking has to be soft, yielding supersymmetric masses around the TeV scale. Therefore, the quest for supersymmetric particles is one of the main topics of the 
current experimental program at the Large Hadron Collider (LHC) at CERN, which is currently exploring the electroweak scale.

The general purpose LHC experiments, ATLAS and CMS, are currently pushing the limits on the masses of the supersymmetric particles to a higher and higher range 314 . However, most of the analyses only hold in the context of the so-called constrained MSSM, where $R$-parity is conserved and where the 105 additional free parameters of the most general ( $R$-parity conserving) version of the MSSM are reduced to a set of four parameters and a sign, assuming an organizing principle based on unification at high energy. In contrast, there exists a vast variety of (nonminimal) supersymmetric models which can be valuable to be investigated both from the point of view of the theorist and the one of the experimentalist.

Studying the hadron-collider phenomenology of these non-minimal models requires the use of Monte Carlo event generators in order to describe both backgrounds and new physics signals. These tools efficiently match a proper modeling of the strong interactions (parton showering, fragmentation and hadronization) with the calculation of matrix elements underlying the considered hard-scattering processes where new physics is expected to appear. For this reason, activities in the field of Monte Carlo simulations have been rather intense over the last twenty years, resulting in many advances through the release of automated tree-level matrix-element generators, such as AlPGen 5, Comix 6, CompHeP/CAlcHeP 7/819, HelaC 10, MadGraph/MadEvent 11/12/13/14|15, Sherpa 16/17 and Whizard 18|19.

Computing predictions for a given new physics theory with the help of the above-mentioned tools requires the implementation of the complete set of associated Feynman rules in these programs. This task has been alleviated by packages such as LANHeP 20|21, FeynRules 22|23|24|25] or SARAH 26|27] which start from the Lagrangian of the theory and export the associated Feynman rules to one or several event generators in an automated fashion. However, whereas all these tools can address the implementation of the Lagrangian in the usual space-time, only the FEYNRULES package is suitable to perform ab initio computations within the superspace formalism $28 \mid 29$, a natural framework for supersymmetric model building.

In a softly broken supersymmetric theory, the field content is embedded into chiral and vector supermultiplets for the matter and gauge sector, respectively, and the Lagrangian is written as a sum of four terms. The first two consist in kinetic terms for the chiral and vector supermultiplets of the model. Assuming renormalizability, these terms are entirely fixed by supersymmetry and gauge invariance and are hence model-independent. The third piece of the Lagrangian describes the interactions among the chiral supermultiplets and is driven by the so-called superpotential, whilst the last piece contains the supersymmetry-breaking terms. These two last parts of the Lagrangian are both model-dependent. In the superspace formalism, the Lagrangian is written in terms of superfields, in contrast to the component field formalism where it is expressed in terms of the usual scalar, fermionic and vector fields of particle physics. The main advantage is related to simpler and very 
compact superfield expressions, in contrast to their component field counterparts. This renders the implementation of softly broken supersymmetric Lagrangians in high-energy physics tools much more straightforward and bypasses many possible sources of mistakes.

The superspace module of FEYNRULES contains a set of functions generating automatically the kinetic terms of the Lagrangian (expressed in terms of superfields). Subsequently, the implementation of any supersymmetric theory in the FEYNRULES package, and therefore in any of the Monte Carlo event generators interfaced to it, consists only in the setting of the superfield content, the model parameters and the gauge symmetries of the theory, together with providing the model dependent parts of the Lagrangian, i.e., the supersymmetry-breaking Lagrangian and the superpotential (the latter being expressed in terms of superfields). Once implemented, the Lagrangian is automatically expanded in terms of component fields by FEYNRULEs, these objects being the ones handled by the Monte Carlo event generators. The Feynman rules are then extracted and the vertices are passed to the different interfaced tools.

This procedure allows for phenomenological investigations of a large class of supersymmetric models whose full implementation in a Monte Carlo event generators was considered too involved so far. Currently, dedicated interfaces exist to the CompHep/Calchep, FeynArts/FormCalc 30131, MadGraph/MadEvent, SherPa and WhizArd programs. In addition, a Universal FeynRules Output (UFO) 32 of the model can be created. In this case, all the model information is exported as a set of PYTHON classes and objects representing particles, parameters and vertices. The produced Pүтнол library contains all the interactions included in the Lagrangian, without any restriction on the allowed Lorentz and/or color structures, in contrast to the other interfaces which reject vertices not compliant with the (hard-coded) structures supported by the corresponding programs. Presently, the UFO format is used by the MADGRAPH (version 5) and the GoSAM 33 generators.

In this work, we present the phenomenological study of collider signatures associated to interaction vertices with exotic color structures. We start by describing how to implement into FEYNRuLes two examples of supersymmetric theories beyond the constrained MSSM in the most automated possible way, to extract the UFO libraries and to use it then with the MADGRAPH Monte Carlo event generator for phenomenological investigations of signatures specific to each model. The choice of the MADGRAPH generator is driven by the color structures which we desire to probe, since among the above-mentioned tools, only MADGRAPH 5 is capable of handling non-standard color or Lorentz structures, using, among others, the flexibility of the UFO model format, which is handled internally by ALOHA 34, an application automatically writing HELAS $35 \mid 36 / 37 / 38$ libraries from Feynman rules corresponding to the model under consideration.

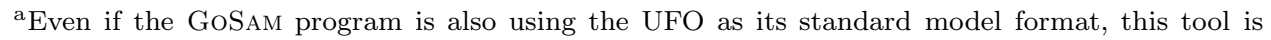
dedicated to next-to-leading order computations, which goes beyond the scope of this work. 
In our first example, we extend the constrained ( $R$-parity conserving) MSSM by allowing superpotential and supersymmetry-breaking Lagrangian terms in which $R$-parity is broken 39 . We choose to focus on a set of particular vertices included in that model which couple three particles lying in the fundamental representation of $S U(3)_{c}$ through a totally antisymmetric tensor of three color (anti)triplet indices. For our second example, we turn to phenomenological investigations of scalar particles lying in the adjoint representation of the $S U(3)_{c}$ gauge group, as predicted by hybrid supersymmetric theories containing representations of both the $N=1$ and $N=2$ superalgebras $40|41| 42|43 / 44| 45 \mid 46$ or by $R$-symmetric supersymmetric models $47 / 48 / 49 / 50$. In those models, the $S U(3)_{c}$ vector supermultiplet of the MSSM is supplemented by a chiral supermultiplet, forming hence a complete gauge supermultiplet of a $N=2$ supersymmetry lying in the adjoint representation of the $S U(3)_{c}$ gauge group. This latter supermultiplet contains a vector field (the gluon), two (two-component) fermions mixing to a Dirac gluino field (in contrast to the Majorana counterpart included in the MSSM) and one scalar particle, commonly dubbed as the sgluon field. Even if pairs of sgluons interact with one or two gluons through the usual covariant derivative of $S U(3)_{c}$, hybrid and $R$-symmetric supersymmetric theories also predict a coupling between a single sgluon and a pair of gluons through a dimension-five operator generated by a loop of squarks, the color part of the interaction vertex being the symmetric tensor of $S U(3)$ 51]. Since the QCD sector is the same in both the $R$-symmetric and hybrid supersymmetric models, we choose to focus, in the following, only on the $R$-symmetric theories for the sake of the example.

Even if we decide to focus on non-minimal softly-broken supersymmetric theories predicting interaction vertices with peculiar color structures, a broad class of extensions of the MSSM contains additional non-renormalizable interactions in the Kähler potential and in the superpotential, leading to non-standard Lorentz structures. These new interactions appear, e.g. in the Higgs sector 52153154 , when we consider possible new physics effects through higher-dimensional operators. These models can be directly implemented into the superspace module of FEYNRULES and consequently exported to Monte Carlo tools for further phenomenological investigations in the same way as for the two examples considered in this work. In this case, Monte Carlo programs containing a hard-coded set of supported Lorentz structures are again not suitable if the relevant structures are too exotic. Once again, the MADGRAPH 5 program, using the UFO and ALOHA, allows for the implementation of any (renormalizable or not) interaction vertex regardless of the complexity of the Lorentz structure.

This paper is organized as follows. In Section 2, we recall the general features of softly-broken supersymmetric Lagrangians and describe the two considered nonminimal supersymmetric theories, namely the MSSM with $R$-parity violation and the minimal $R$-symmetric supersymmetric model, and their implementation within the superspace module of FEYNRULES starting from the existing MSSM implemen- 
tation. In Section 3, we present the extraction of the UFO model format and its linking to MADGRAPH 5, whilst Section 4 focuses on the adopted benchmark scenarios and the description of the high energy physics process of interest. Section 5 is dedicated to our phenomenological analyses and our conclusions are finally given in Section 6 ,

\section{Softly-broken supersymmetric theories and their implementation in FeynRules}

In this Section, we describe the main features of softly-broken supersymmetric theories and how to implement the corresponding Lagrangians into the superspace module of FEYNRulEs. For further information, we refer to the manual of FEYNRULES 22 and to the details of its superspace module 25 . We then describe the two non-minimal supersymmetric models which we propose to investigate, i.e., the Minimal Supersymmetric Model with $R$-parity violation and the Minimal $R$-symmetric Supersymmetric Standard Model, and finally address their implementation within FEYNRULES.

\subsection{A generic supersymmetric theory in the superspace formalism}

\subsubsection{Superfields}

Supersymmetric theories are more elegantly formulated within the superspace formalism 28|29. Superspace is an extension of the ordinary space-time where we supplement the usual space-time coordinates $x^{\mu}$ by the variables $\theta$ and $\bar{\theta}$, transforming as two-component Weyl fermions with opposite chirality (forming thus a Majorana spinor $\left.\left(\theta^{\alpha}, \bar{\theta}_{\dot{\alpha}}\right)\right)$. A superfield $\Phi$ is any function of the superspace coordinates and can generally expanded as a (finite) Taylor series with respect to the variables $\theta$ and $\bar{\theta}$,

$$
\begin{gathered}
\Phi(x, \theta, \bar{\theta})=z(x)+\theta \cdot \xi(x)+\bar{\theta} \cdot \bar{\zeta}(x)+\theta \cdot \theta f(x)+\bar{\theta} \cdot \bar{\theta} g(x)+\theta \sigma^{\mu} \bar{\theta} v_{\mu}(x) \\
+\bar{\theta} \cdot \bar{\theta} \theta \cdot \omega(x)+\theta \cdot \theta \bar{\theta} \cdot \bar{\rho}(x)+\theta \cdot \theta \bar{\theta} \cdot \bar{\theta} d(x)
\end{gathered}
$$

The various coefficients of this expansion are the component fields of the superfield $\Phi$ and form a so-called supermultiplet. The fields $z, f, g$ and $d$ are thus complex scalar fields, the spinors $\xi, \zeta, \omega$ and $\rho$ are complex Weyl fermions and $v_{\mu}$ is a complex vector field. In the expression above, we have introduced the four-vector built from the Pauli matrices $\sigma^{\mu}$. All the conventions, and in particular those related to spinors (invariant products, position of the indices, ...), strictly follow those presented in Ref. 25 and Ref. 55 .

The unconstrained superfield $\Phi$ of Eq. (1) provides a reducible representation of the supersymmetric algebra, the number of degrees of freedom embedded in the expansion of $\Phi$ being too large with respect to those included in the supermultiplets representing the $N=1$ supersymmetric algebra. Consequently, constraints are imposed on the generic superfield $\Phi$ so that the number of independent component 
Benjamin Fuks

fields is drastically reduced. The two types of superfields necessary to construct most of the phenomenologically relevant supersymmetric theories are chiral superfields, which include one complex scalar and one two-component fermionic component, and vector superfields whose component fields are one real massless vector field and the associated Majorana fermion.

Left and right-handed chiral superfields $\Phi_{L}$ and $\Phi_{R}$ satisfy the constraints

$$
\bar{D}_{\dot{\alpha}} \Phi_{L}(x, \theta, \bar{\theta})=0 \quad \text { and } \quad D_{\alpha} \Phi_{R}(x, \theta, \bar{\theta})=0,
$$

where the superderivatives $D_{\alpha}$ and $\bar{D}_{\dot{\alpha}}$ read, in our conventions $\underline{55}$,

$$
D_{\alpha}=\frac{\partial}{\partial \theta^{\alpha}}-i \sigma_{\alpha \dot{\alpha}}^{\mu} \bar{\theta}^{\dot{\alpha}} \partial_{\mu} \quad \text { and } \quad \bar{D}_{\dot{\alpha}}=\frac{\partial}{\partial \bar{\theta}^{\dot{\alpha}}}-i \theta^{\alpha} \sigma_{\alpha \dot{\alpha}}^{\mu} \partial_{\mu} .
$$

Under the constraints of Eq. (2), the number of degrees of freedom included in the expansion of the superfields $\Phi_{L}$ and $\Phi_{R}$ with respect to the variables $\theta$ and $\bar{\theta}$ match those of the matter supermultiplets. The expansions of these superfields are indeed given by

$$
\begin{aligned}
\Phi_{L}(y, \theta) & =\phi(y)+\sqrt{2} \theta \cdot \psi(y)-\theta \cdot \theta F(y), \\
\Phi_{R}\left(y^{\dagger}, \bar{\theta}\right) & =\phi\left(y^{\dagger}\right)+\sqrt{2} \bar{\theta} \cdot \bar{\psi}\left(y^{\dagger}\right)-\bar{\theta} \cdot \bar{\theta} F\left(y^{\dagger}\right) .
\end{aligned}
$$

In these two equations, we have introduced the reduced variable $y^{\mu}=x^{\mu}-i \theta \sigma^{\mu} \bar{\theta}$. As stated above, the expansion of the superfields $\Phi_{L}$ and $\Phi_{R}$ includes hence a complex scalar field $\phi$, a Weyl fermion $\psi$ and an auxiliary additional scalar field $F$, which is necessary to restore equality between the number of bosonic and fermionic degrees of freedom for off-shell supermultiplets.

Chiral superfields can be declared in FeynRules model files in a way similar to the one employed for the declaration of ordinary fields. This extends the original FEYNARTS conventions to the superfield level. Superfields with the same quantum numbers are collected into classes and each class is implemented as a list of Mathematic $\sqrt{b}$ replacement rules. For example, a left-handed superfield $\Phi$ could be implemented as

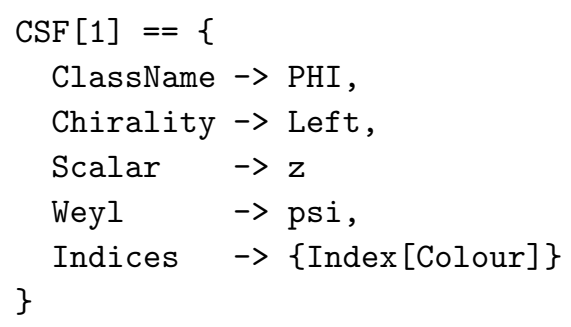

This declares simultaneously a left-handed chiral superfield CSF [1] represented by the variable PHI and the associated right-handed chiral superfield $\Phi^{\dagger}$. The latter can then be used in FEYNRULES through the (automatically created) symbol PHIbar. In

b MAThematica is a registered trademark of Wolfram Research Inc. 
addition, the implemented superfield carries an index labeled by Colour, illustrating the fact that it lies in a non-trivial representation of the QCD gauge group (see below). The scalar and fermionic component fields of $\Phi$ are represented by the symbols $z$ and psi, respectively, and it is important to note that these two fields must be independently declared in the FeynRules model file. In contrast, the auxiliary $F$-field is internally handled and the user does not have to take care of it. For instance, the scalar component field could be included in the model file as

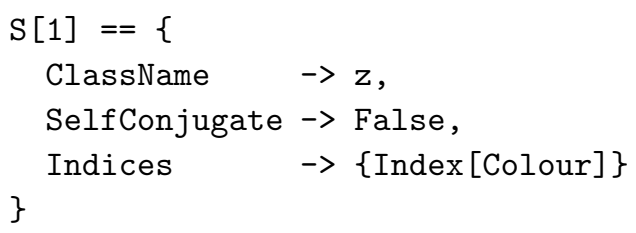

We emphasize that the options, such as Indices, which are shared by the component field class and the superfield class must be identical. A complete description of the particle and superfield classes and properties can be found in Ref. 22 and Ref. 25.

Vector superfields describe the representations of the $N=1$ supersymmetric algebra containing one massless gauge boson together with the corresponding fermionic degree of freedom. These multiplets are embedded into vector superfields defined by the reality condition

$$
V=V^{\dagger}
$$

However, this last condition leads to numerous non-physical fields which have to be integrated out. In a suitable gauge, the Wess-Zumino gauge, these unphysical components are eliminated already at the level of the expansion of the superfield $V$ in terms of the variables $\theta$ and $\bar{\theta}$,

$$
V=\theta \sigma^{\mu} \bar{\theta} v_{\mu}+i \theta \cdot \theta \bar{\theta} \cdot \bar{\lambda}-i \bar{\theta} \cdot \bar{\theta} \theta \cdot \lambda+\frac{1}{2} \theta \cdot \theta \bar{\theta} \cdot \bar{\theta} D
$$

This gauge choice is adopted in the rest of this paper. The component fields included in the expansion of the superfield $V$ are a real vector field $v_{\mu}$, a Majorana spinor $\left(\lambda_{\alpha}, \bar{\lambda}^{\dot{\alpha}}\right)$ and an additional auxiliary real scalar field $D$ ensuring equal numbers of fermionic and bosonic degrees of freedom when going off-shell.

This superfield can be declared in FeYnRULes model files in a similar fashion as for the chiral superfield. Taking the example of the QCD gauge group, one could have

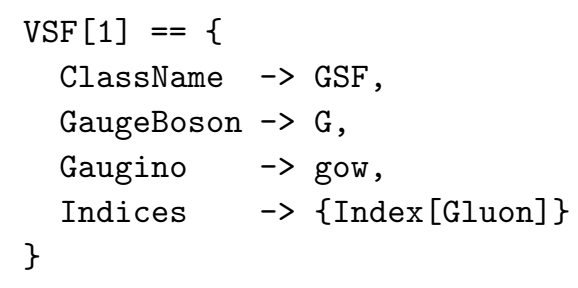


Benjamin Fuks

This list of MAthematica replacement rules assigns the symbol GSF to the vector superfield VSF [1] and defines the related physical components fields by the variables G, representing the gluon, and gow, corresponding to the two-component gluino spinor. As for the chiral superfield case, both the gluon and gluino fields must be properly declared in the model file and the auxiliary $D$-field is treated internally by FeynRules. As vector superfields and their component fields are associated to the mediation of the gauge interactions, they naturally lie in the adjoint representation of the gauge group. This is emphasized by the presence of an index denoted Gluon among the properties of the superfield VSF [1], and this index is related to the adjoint representation of $S U(3)_{c}$ once this group is defined. This last task could be performed through the declaration

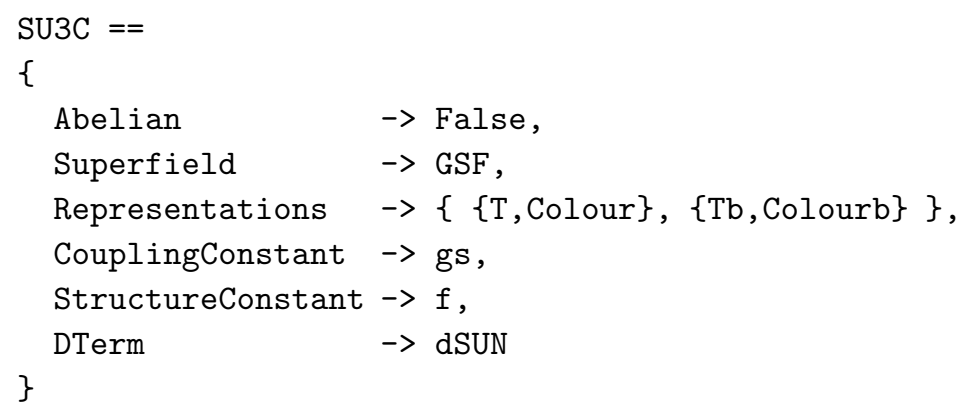

This last MATHematica command declares a variable SU3C representing the $S U(3)_{c}$ gauge group and associated to the superfield GSF through the Superfield option of the gauge group class. This mapping internally defines the indices carried by the superfield GSF as those related to the adjoint representation of the gauge group. In addition, two other representations are declared, the symbols Colour and Colourb being the fundamental and anti-fundamental representation indices and $\mathrm{T}$ and $\mathrm{Tb}$ the corresponding representation matrices. The declaration of the gauge group also assigns the symbol gs to the strong coupling constant and dSUN and $f$ to the symmetric tensor and antisymmetric structure constants of $S U(3)$, respectively. This allows to use consistently build-in FEYNRULEs functions computing automatically, e.g., the superfield strength tensor or the chiral superfield kinetic terms.

\subsubsection{Lagrangians}

A generic supersymmetric model is defined by fixing the superfield content and the gauge symmetries of the theory. We consider a simple gauge group $G$ and denote by $g$ the related gauge coupling constant. We introduce a set of irreducible representations $\mathcal{R}$ of the corresponding Lie algebra $\mathfrak{g}$ which are spanned by the

${ }^{\mathrm{c}}$ Strong interactions play a special role in Monte Carlo programs related to the simulation of hadron collisions. Consequently, the names of the quantities related to the QCD gauge group, such as the index related to its adjoint representation, follow the strict conventions depicted in Ref. 22 
hermitian matrices $T^{a}$, and to each matrix, associate a vector superfield $V^{a}$. The matter sector consists in a set of matter supermultiplets described by left-handed chiral superfields $\Phi^{i}$ lying in the representation $\mathcal{R}^{i}$ of $\mathfrak{g}$, whilst the gauge sector is described by a vector superfield $V=V^{a} T_{a}$. In the case of a semi-simple gauge group, i.e., when the gauge group consists in a direct product of several simple gauge groups, it is sufficient to generalize the previous setup by associating one vector superfield $V$ to each direct factor $G^{j}$ of the gauge group. The chiral superfields of the theory then lie in a given representation of the algebras corresponding to each of the $G^{j}$ groups.

In their most general (non-renormalizable) version, softly-broken supersymmetric Lagrangians can be expressed entirely with the help of three fundamental functions of the superfields of the theory $56|57| 58 \mid 59$, namely the Kähler potential $K$, the superpotential $W$ and the gauge kinetic function $h_{a b}$, given together with the soft supersymmetry breaking terms. The Kähler potential is a real function of the chiral and anti-chiral superfield content of the theory and contains kinetic terms describing its dynamics. The superpotential is an holomorphic function of the chiral superfields and describes their interactions not driven by the Nother procedure leading to the covariantization of the Lagrangian both with respect to gauge and supersymmetric transformations. The gauge kinetic function, symmetric in the two adjoint gauge indices $a$ and $b$, is also an holomorphic function of the chiral superfields, and addresses the kinetic terms related to the vector supermultiplets of the theory. Let us note that the Kähler potential and the gauge kinetic function have a very simple form if one assumes renormalizability. In this case, the Lagrangian is much simpler and its implementation in FEYNRULES can be automated (see below). We however first turn to the most general case.

Putting everything together, (non-renormalizable) softly-broken supersymmetric Lagrangian reads

$$
\begin{aligned}
\mathcal{L}=\int & \mathrm{d}^{2} \theta \mathrm{d}^{2} \bar{\theta} \frac{1}{2}\left[K\left(\Phi, \Phi^{\dagger} e^{-2 g V}\right)+K\left(e^{-2 g V} \Phi, \Phi^{\dagger}\right)\right] \\
& +\frac{1}{16 g^{2}} \int \mathrm{d}^{2} \theta h_{a b}(\Phi) W^{a \alpha} W_{\alpha}^{b}+\frac{1}{16 g^{2}} \int \mathrm{d}^{2} \bar{\theta} h_{a b}^{\star}\left(\Phi^{\dagger}\right) \bar{W}_{\dot{\alpha}}^{a} \bar{W}^{b \dot{\alpha}} \\
& +\int \mathrm{d}^{2} \theta W(\Phi)+\int \mathrm{d}^{2} \bar{\theta} W^{\star}\left(\Phi^{\dagger}\right)+\mathcal{L}_{\text {soft }},
\end{aligned}
$$

where an implicit sum over the chiral and gauge superfields $\Phi$ and $V$ is understood, if relevant, and where $W^{\star}$ and $h^{\star}$ are the functions conjugate to $W$ and $h$, respectively. In this last equation, we have introduced the soft-supersymmetry breaking Lagrangian $\mathcal{L}_{\text {soft }}$ and the spinorial superfield strength tensors. In the conventions of Ref. $\frac{55}{5}$ they read,

$$
W_{\alpha}=-\frac{1}{4} \bar{D} \cdot \bar{D} e^{2 g V} D_{\alpha} e^{-2 g V} \quad \text { and } \quad \bar{W}_{\dot{\alpha}}=-\frac{1}{4} D \cdot D e^{-2 g V} \bar{D}_{\dot{\alpha}} e^{2 g V}
$$

where the superderivatives have been defined in Eq. (3). 
The general Lagrangian of Eq. (77) can be included in a straightforward manner in FEynRules model files using the strengths of the superspace module. One possible implementation would proceed in two steps. Firstly, the superpotential, expressed in terms of superfields, is stored in the variable SuperPot and the supersymmetrybreaking Lagrangian, generally expressed in terms of component fieldsd, is implemented in the variable LSoft. Secondly, for the kinetic part of the Lagrangian, we define the quantities

$$
\Omega=\frac{1}{2}\left[K\left(\Phi, \Phi^{\dagger} e^{-2 g V}\right)+K\left(e^{-2 g V} \Phi, \Phi^{\dagger}\right)\right] \text { and } \quad \rho=h_{a b}(\Phi) W^{a \alpha} W_{\alpha}^{b},
$$

and implement them (in terms of superfields) in the FEYNRULEs model file within the variables Omega and rho, respectively. An implementation of the Lagrangian of Eq. (7) is finally given by

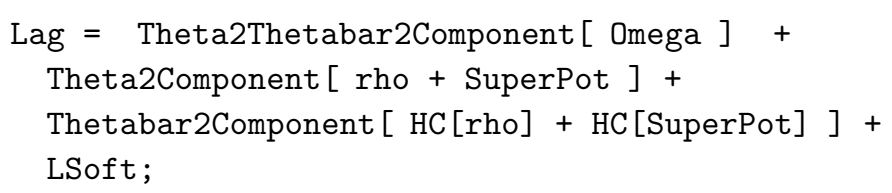

In this set of commands, we have introduced the FeynRules functions Theta2Component, Thetabar2Component, Theta2Thetabar2Component allowing to perform the two- and four-dimensional integrations over the Grassmann variables $\theta$ and $\bar{\theta}$, together with the function $\mathrm{HC}$ which transforms an expression into the hermitian conjugate counterpart. Let us note that the left-handed and right-handed superfield strength tensors appearing in the definition of $\rho$ can be cast within the functions SuperfieldStrengthL and SuperfieldStrengthR. We refer to Ref. 25 for more details.

The Lagrangian density of Eq. (7) still depends on the unphysical $F$ and $D$ degrees of freedom of the theory and is expressed in terms of Weyl fermions. In contrast, all Monte Carlo event generators which could be used to investigate the phenomenology of any softly-broken supersymmetric model require the usage of four-component Majorana and Dirac (and not two-component) fermions and physical fields. The FEynRuLES package provides a couple of automated functions allowing to render the Lagrangian compliant with the requirements of the Monte Carlo programs.

Firstly, the unphysical fields can be integrated out of the Lagrangian by inserting the solution of their equations of motion. This can be performed by employing the functions SolveEqMotionD and SolveEqMotionF for the $D$ - and $F$-terms, respectively,

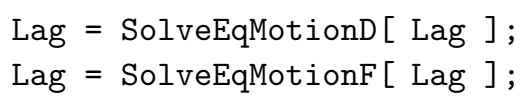

${ }^{\mathrm{d}}$ For a soft-supersymmetry breaking Lagrangian defined in terms of superfields and integration over the superspace, we refer to Section 2.3 
Secondly, the WeylComponents option of the four-component fermion particle class allows to link a four-component fermion to its two-component counterparts. Set up in this way, FEYNRULES replaces any occurrence of a product of Weyl spinors by the corresponding product of four-component spinors. This task is achieved through the automated function WeylToDirac. However, before performing these transformations, care must be taken with the gauge group representations in which the considered fields lie. Indeed, supersymmetric field theories are built only with the help of left-handed chiral superfields. Subsequently, the right-handed parts of the four-component fermions are embedded into the corresponding charge-conjugate left-handed chiral superfield, lying thus in the complex conjugate representation of the gauge group. Mapping Weyl fermions to Dirac and Majorana fermions requires then a specific treatment. Taking the example of the QCD gauge group implemented above, it is sufficient to render the indices Colour and Colourb equal and use the properties of the representation matrices $\bar{T}=-T^{*}=-T^{t}$ to get rid of any track of the anti-fundamental representation of $S U(3)_{c}, T$ being the fundamental and $-T^{*}$ the anti-fundamental representation matrices, i.e., the complex conjugate representation. Hence, a possible implementation would be

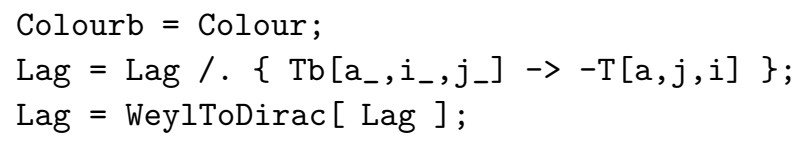

For renormalizable theories, the Kähler potential and the gauge kinetic functions take a trivial form

$$
K\left(\Phi, \Phi^{\dagger}\right)=\Phi^{\dagger} \Phi \quad \text { and } \quad h_{a b}(\Phi)=\delta_{a b},
$$

and the Lagrangian of Eq. (77) simplifies. The complete model is then described by the superpotential and the soft-supersymmetry breaking terms alone, the $\Omega$ and $\rho$ variables being fully fixed by Eq. (10). In this case, these quantities can be computed automatically with the help of the CSFKineticTerms and VSFKineticTerms functions of FEYNRULES, the only task left to the user being the implementation of the superfield content of the theory, the superpotential and the soft-supersymmetry breaking Lagrangian. This renders the implementation of any softly-broken supersymmetric theory in FeynRules, and therefore in the Monte Carlo programs interfaced to it, almost entirely automated. The two examples investigated in this work follow that approach, the tasks related to programming which are left to the user being therefore minimal.

\subsection{The Minimal Supersymmetric Standard Model with R-parity violation}

\subsubsection{Theoretical framework}

The MSSM is the simplest supersymmetric model, resulting from a direct supersymmetrization of the Standard Model 12. In the most general form allowed 
by gauge invariance and renormalizability, the superpotential includes dangerous baryon-number $(B)$ and lepton-number $(L)$ violating interactions predicting, e.g., fast proton decays, atomic parity violation or large flavor-changing neutral currents 39. To circumvent this issue, a discrete symmetry is imposed, namely $R$-parity, which forbids all the problematic terms.

However, neither $B$ nor $L$ conservations are fundamental $[60$ and there is no strong theoretical motivation to forbid the $R$-parity violating terms of the MSSM superpotential. One could rather assume their existence and derive strong experimental constraints on the corresponding coupling strengths. In general, among all the possible $R$-parity violating terms, data tends to show a single coupling dominance effect, i.e., only one single $R$-parity coupling can be non-negligible at a time 6162

The MSSM is based on the same semi-simple gauge group as the Standard Model, $G_{M S S M} \equiv S U(3)_{c} \times S U(2)_{L} \times U(1)_{Y}$. We associate one vector superfield to each of the direct factors of the gauge group. These vector superfields, labeled by $V_{G}, V_{W}$ and $V_{Y}$, lie in the adjoint representation of the corresponding simple Lie group and are singlets under all the other factors of $G_{M S S M}$. They are given, together with their representations under $G_{M S S M}$, by

$$
\begin{aligned}
S U(3)_{c} & \Leftrightarrow V_{G}=(\underset{\sim}{\mathbf{8}}, \underset{\sim}{\mathbf{1}}, 0), \\
S U(2)_{L} & \Leftrightarrow V_{W}=(\underset{\sim}{\mathbf{1}}, \underset{\sim}{\mathbf{3}}, 0), \\
U(1)_{Y} & \Leftrightarrow V_{B}=(\underset{\sim}{\mathbf{1}}, \underset{\sim}{\mathbf{1}}, 0) .
\end{aligned}
$$

The set of physical component fields includes, in addition to the Standard Model gauge bosons, the gaugino partners, as shown in Table 1

Table 1. The superfields included MSSM gauge sector. The gauge boson and gaugino components are given together with the representations under the $S U(3)_{c} \times S U(2)_{L} \times U(1)_{Y}$ gauge group.

\begin{tabular}{|c||c|c|c|}
\hline Superfield & Gauge boson & Gaugino & Representation \\
\hline \hline$V_{B}$ & $B_{\mu}$ & $\widetilde{B}$ & $(\mathbf{1}, \mathbf{1}, 0)$ \\
$V_{W}$ & $W_{\mu}$ & $\widetilde{W}$ & $(\mathbf{1}, \mathbf{3}, 0)$ \\
$V_{G}$ & $g_{\mu}$ & $\widetilde{g}$ & $(\mathbf{8}, \mathbf{1}, 0)$ \\
\hline
\end{tabular}

The chiral sector of the theory consists of three generations of Standard Model quarks and leptons embedded into three generations of chiral supermultiplets, together with their squark and slepton partners,

$$
\begin{array}{lll}
Q_{L}^{i} & =\left(\underset{\sim}{(\mathbf{3}}, \underset{\sim}{\mathbf{2}}, \frac{1}{6}\right) \quad, \quad U_{R}^{i}=\left(\underset{\sim}{\overline{\mathbf{3}}}, \underset{\sim}{\mathbf{1}},-\frac{2}{3}\right) \quad, \quad D_{R}^{i}=\left(\underset{\sim}{\overline{\mathbf{3}}}, \underset{\sim}{\mathbf{1}}, \frac{1}{3}\right) \\
L_{L}^{i}=\left(\underset{\sim}{\mathbf{1}}, \underset{\sim}{\mathbf{2}},-\frac{1}{2}\right) & , \quad E_{R}^{i}=(\underset{\sim}{\mathbf{1}}, \underset{\sim}{\mathbf{1}}, 1) \quad, \quad V_{R}^{i}=(\underset{\sim}{\mathbf{1}}, \underset{\sim}{\mathbf{1}}, 0),
\end{array}
$$


where $i$ stands for a generation index and where we have indicated the representations of the different superfields under the MSSM gauge group. Let us note that since supersymmetric theories must be built only with left-handed (and not righthanded) chiral superfields, the associated superfields include the charge-conjugate right-handed Standard Model fermions and lie thus in the corresponding complexconjugate representations of $G_{M S S M}$. For completeness, we have included the righthanded neutrino superfields. However, they will be kept non-interacting with any other superfield, even at the level of the superpotential.

In addition, (s)fermion superfields are supplemented with two Higgs chiral supermultiplets, embedding thus two $S U(2)_{L}$ doublets of scalar Higgs fields accompanied by two doublets of their fermionic Higgsino partners,

$$
H_{D}=\left(\underset{\sim}{\mathbf{1}}, \underset{\sim}{\mathbf{2}},-\frac{1}{2}\right) \quad, \quad H_{U}=\left(\underset{\sim}{\mathbf{1}}, \mathbf{2}, \frac{1}{2}\right) .
$$

This leads to electroweak symmetry breaking without introducing chiral anomalies and to mass generation for both the up-type and down-type fermions. The component fields included in the full matter sector can be found in Table 2 .

Table 2. The superfields included in the MSSM chiral sector. The scalar and two-component spinor components are given together with the representations under the $S U(3)_{c} \times S U(2)_{L} \times U(1)_{Y}$ gauge group. The superscript $c$ denotes charge conjugation.

\begin{tabular}{|c||c|c|c|}
\hline Superfield & Fermion & Scalar & Representation \\
\hline \hline$Q_{L}^{i}$ & $q_{L}^{i}=\left(\begin{array}{c}u_{L}^{i} \\
d_{L}^{i}\end{array}\right)$ & $\tilde{q}_{L}^{i}=\left(\begin{array}{c}\tilde{u}_{L}^{i} \\
\tilde{d}_{L}^{i}\end{array}\right)$ & $\left(\mathbf{3}, \mathbf{2}, \frac{1}{6}\right)$ \\
$U_{R}^{i}$ & $u_{R}^{i c}$ \\
$D_{R}^{i}$ & $d_{R}^{i c}$ & $\tilde{u}_{R}^{i \dagger}$ & $\left(\begin{array}{c}\left(\overline{\mathbf{3}}, \mathbf{1},-\frac{2}{3}\right) \\
\left(\overline{\mathbf{3}}, \mathbf{1}, \frac{1}{3}\right)\end{array}\right.$ \\
\hline$L_{R}^{i \dagger}$ & $\ell_{L}^{i}=\left(\begin{array}{c}\nu_{L}^{i} \\
e_{L}^{i}\end{array}\right)$ & $\tilde{\ell}_{L}^{i}=\left(\begin{array}{c}\tilde{\nu}_{L}^{i} \\
\tilde{e}_{L}^{i}\end{array}\right)$ & $\left(\mathbf{1}, \mathbf{2},-\frac{1}{2}\right)$ \\
$E_{R}^{i}$ & $e_{R}^{i c}$ & $\tilde{e}_{R}^{i \dagger}$ & $(\mathbf{1}, \mathbf{1}, 1)$ \\
$V_{R}^{i}$ & $\nu_{R}^{i c}$ & $\tilde{\nu}_{R}^{i \dagger}$ & $(\mathbf{1}, \mathbf{1}, 0)$ \\
\hline$H_{D}$ & $\widetilde{H}_{d}=\left(\begin{array}{c}\widetilde{H}_{d}^{0} \\
\widetilde{H}_{d}^{-}\end{array}\right)$ & $H_{d}=\left(\begin{array}{c}H_{d}^{0} \\
H_{d}^{-}\end{array}\right)$ & $\left(\mathbf{1}, \mathbf{2},-\frac{1}{2}\right)$ \\
& & \\
$H_{U}$ & $\widetilde{H}_{u}=\left(\begin{array}{l}\widetilde{H}_{u}^{+} \\
\widetilde{H}_{u}^{0}\end{array}\right)$ & $H_{u}=\left(\begin{array}{l}H_{u}^{+} \\
H_{u}^{0}\end{array}\right)$ & $\left(\mathbf{1}, \mathbf{2}, \frac{1}{2}\right)$ \\
\hline
\end{tabular}


As stated in Section 2.1 the kinetic and gauge interaction terms of the chiral and vector superfields are entirely fixed by gauge invariance and supersymmetry. Considering the simplest model with trivial Kähler potential and gauge kinetic function (see Eq. (10)), the kinetic Lagrangian reads,

$$
\begin{aligned}
\mathcal{L}= & \sum_{k=S U(3)_{c}, S U(2)_{L}, U(1)_{Y}}\left(\int \mathrm{d}^{2} \theta \frac{W_{k}^{\alpha} W_{\alpha}^{k}}{16 g^{2}}+\int \mathrm{d}^{2} \bar{\theta} \frac{\bar{W}_{k \dot{\alpha}} \bar{W}_{\dot{\alpha}}^{k}}{16 g^{2}}\right) \\
& +\sum_{\Phi=\text { chiral content }} \int \mathrm{d}^{2} \theta \mathrm{d}^{2} \bar{\theta} \Phi^{\dagger}\left(e^{-Y g^{\prime} V_{B}} e^{-2 g_{w} \tilde{V}_{W}} e^{-2 g_{s} \tilde{V}_{G}}\right) \Phi
\end{aligned}
$$

where the superfield strength tensors have been defined in Eq. (8). We have introduced the non-Abelian vector superfields $\tilde{V}_{W}=V_{W^{k}} T_{k}, \tilde{V}_{G}=V_{G^{a}} T_{a}$, where the $S U(2)_{L}$ and $S U(3)_{c}$ generators $T_{k}$ and $T_{a}$ are taken, for each term in the sum, in the representation appropriate to the considered chiral superfield $\Phi$. The three gauge coupling constants are denoted by $g^{\prime}, g_{w}$ and $g_{s}$.

Non-gauge interactions among the chiral superfields introduced in Eq. (12) and Eq. (13) are driven by the superpotential. The superpotential of our model is taken as the one of the $R$-parity conserving MSSM, denoted by $W_{R P C}$, supplemented by the so-called $\lambda$ terms,

$$
\begin{aligned}
W(\Phi)= & W_{R P C} \\
& +\frac{1}{2} \lambda_{i j k} L_{L}^{i} \cdot L_{L}^{j} E_{R}^{k}+\lambda_{i j k}^{\prime} L_{L}^{i} \cdot Q_{L}^{j \ell} D_{R \ell}^{k}+\frac{1}{2} \lambda_{i j k}^{\prime \prime} \epsilon^{\ell m n} U_{R \ell}^{i} D_{R m}^{j} D_{R n}^{k} .
\end{aligned}
$$

In this expression, we have explicitly indicated flavor indices $(i, j$ and $k$ ), (anti)fundamental color indices $(\ell, m, n)$ and the $S U(2)$ invariant product (as a '.').

Similarly, additional $R$-parity violating trilinear scalar interactions derived from the form of the superpotential can be included in the soft-supersymmetry breaking Lagrangian,

$$
L_{\mathrm{soft}}=L_{\mathrm{s}, R P C}-\frac{1}{2} T_{i j k} \tilde{l}_{L}^{i} \tilde{l}_{L}^{j} \tilde{e}_{R}^{k}-T_{i j k}^{\prime} \tilde{l}_{L}^{i} \cdot \tilde{q}_{L}^{j \ell} \tilde{d}_{R \ell}^{k}-\frac{1}{2} T_{i j k}^{\prime \prime} \epsilon^{\ell m n} \tilde{u}_{R \ell}^{i} \tilde{d}_{R m}^{j} \tilde{d}_{R n}^{k}+\text { h.c. },
$$

where the coupling strengths are included in the $T, T^{\prime}$ and $T^{\prime \prime}$ parameters and the Lagrangian $L_{\mathrm{s}, R P C}$ is the $R$-parity conserving MSSM soft-supersymmetry breaking Lagrangian.

\subsubsection{Implementation in FeynRules}

In the $R$-parity violating scenario described above, the superfield content of the theory is identical to the one of the usual $R$-parity conserving MSSM. Moreover, all particle mixings occurring after electroweak symmetry breaking are also left unchanged, since the additional terms do not generate further mixing. Hence, the 
relations linking the gauge- and the mass-eigenstate bases are left unchanged with respect to the $R$-parity conserving MSSM. Therefore, implementing the considered model into FEYNRules can be performed very efficiently with a minimal effort. Implementing all the modifications in a file labeled rpv.fr, it is then sufficient to load this file in FEYNRULES simultaneously with the built-in MSSM model file, denoted by mssm.fr,

LoadModel ["mssm.fr", "rpv.fr"];

For details on the mssm.fr file, which can be downloaded from the FEYNRULES online model database, we refer to Ref. 25 ,

The file rpv.fr includes, on the one hand, the definition of the $R$-parity violating parameters $\lambda, \lambda^{\prime}, \lambda^{\prime \prime}, T, T^{\prime}$ and $T^{\prime \prime}$. This follows the standard rules for implementing instances of the particle class (see the FEYNRULES manual). On the other hand, this file contains the implementation of the model Lagrangian. The kinetic terms presented in Eq. (14) are directly implemented using the automated functions CSFKineticTerms and VSFKineticTerms,

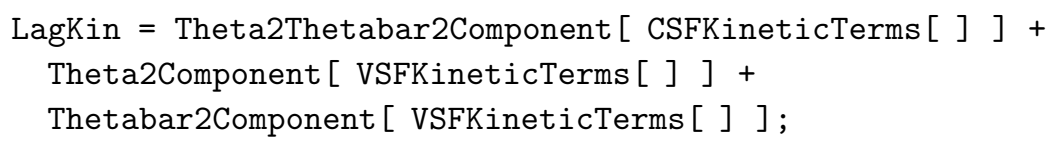

The $R$-parity conserving MSSM superpotential is stored in the file mssm.fr in the variable SPot. The complete Lagrangian containing the interactions driven by the superpotential of Eq. (15) can then be implemented in the rpv.fr file as

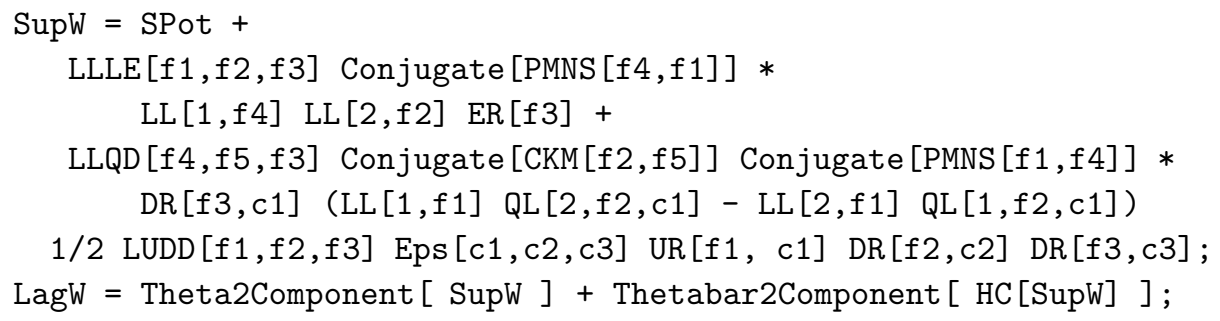

In the MATHEMATICA commands above, the symbols LLLE, LLQD and LUDD stand for the $\lambda, \lambda^{\prime}$ and $\lambda^{\prime \prime}$ parameters of the superpotential, Eps for the fully antisymmetric tensor of rank three and LL, ER, QL, UR and DR for the chiral superfields $L_{L}, E_{R}$, $Q_{L}, U_{R}$ and $D_{R}$. For the first and second terms, the CKM and PMNS matrices are explicitly included. This allows to compensate further field redefinitions included in the model file (see Ref. 25] for more information).

On the same footings, assuming that the soft-supersymmetry breaking Lagrangian associated to the MSSM with $R$-parity conservation is implemented in the variable LS, (see mssm.fr), the full supersymmetry-breaking Lagrangian is implemented from the expression of Eq. (16),

Tsoft $=\operatorname{TLLE}[\mathrm{f} 1, \mathrm{f} 2, \mathrm{f} 3]$ Conjugate $[\mathrm{PMNS}[\mathrm{f} 4, \mathrm{f} 1]] *$ 


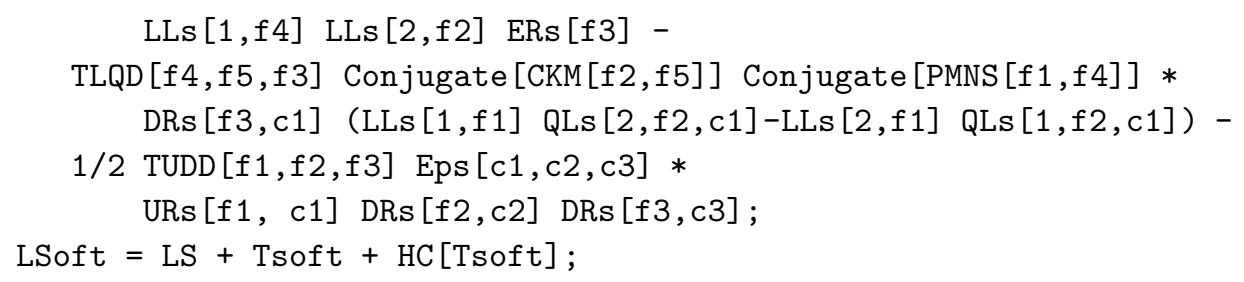

where the symbols TLLE, TLQD and TUDD stand for the $T, T^{\prime}$ and $T^{\prime \prime}$ parameters of the soft-supersymmetry breaking Lagrangian and LLS, ERs, QLs, URs and DRs for the scalar component of the $L_{L}, E_{R}, Q_{L}, U_{R}$ and $D_{R}$ superfields, respectively. Concerning the presence of the CKM and PMNS matrices, we again refer to Ref. 25 .

The complete model Lagrangian is thus given by

$$
\text { Lag }=\text { LagKin }+ \text { LagW }+ \text { LSoft }
$$

In order to render this Lagrangian Lag compliant with the requirements of the Monte Carlo programs which are aimed to be used, the auxiliary $F$ - and $D$-fields must be integrated out. This can be performed with the help of the SolveEqMotionD and SolveEqMotionF commands,

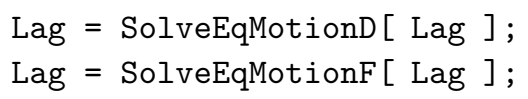

In addition, all Weyl fermions must be replaced in terms of their four-component counterparts,

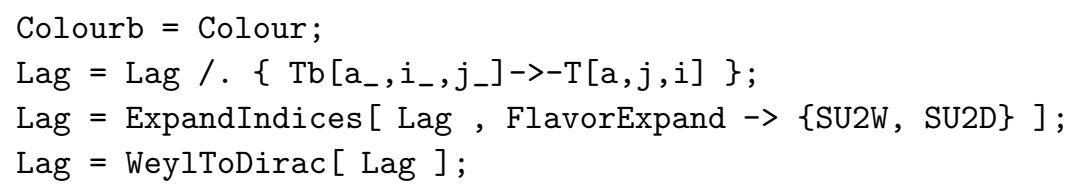

The set of commands above first maps the matrices of the anti-fundamental representation of $S U(3)_{c}$ to those related to the fundamental one (see section 2.1.2), and then performs an expansion of all the indices, fundamental or adjoint, related to the $S U(2)_{L}$ group. This procedure forces the $S U(2)_{L}$ field rotations from the gauge basis to the mass basis, which is necessary for the function WeylToDirac to correctly perform the translation to Dirac and Majorana fermions 25. Let us note that in the current version of FEYNRULES, the indices SU2W, SU2D and Colourb are non standard. Therefore, the manipulations above have to be performed for any model. However, in future versions of the code, the issue of automating this step will be addressed. 


\subsection{The Minimal R-symmetric Supersymmetric Standard Model}

\subsubsection{Theoretical framework}

The supersymmetry algebra naturally contains a continuous $R$-symmetry and there is a large class of mechanisms leading to supersymmetry breaking without breaking this $R$-symmetry 6364 . Among the major consequences of the conservation of this symmetry, Majorana gaugino masses are forbidden. Therefore, phenomenologically viable models requires the pairing of each gaugino with the fermionic component of a new chiral superfield to form a massive Dirac fermion $65[66[67$.

The preservation of the $R$-symmetry also forbids bilinear Higgs mixing term of the superpotential and soft-supersymmetry breaking trilinear scalar interactions. Therefore, phenomenological viability requires the introduction of another set of chiral superfields, the $R$-partners of the Higgs superfields. Mixing terms between the Higgs and these new superfields can be included in the superpotential. Consequently, this renders the Higgsino fields massive and restores agreement with the experimental non-observation of a massless Higgsino field.

Finally, an unbroken $R$-symmetry also ensures that most of the dangerous flavorchanging operators which could appear in generic supersymmetric theories are naturally forbidden.

Starting from the gauge group $G_{M S S M} \equiv S U(3)_{c} \times S U(2)_{L} \times U(1)_{Y}$ and the MSSM superfield content presented in Tables 1 and 2, we add the three chiral superfields $\Phi_{B}, \Phi_{W}$ and $\Phi_{G}$ to the theory. They lie in the adjoint representation of the relevant gauge group,

$$
\Phi_{B}=(\underset{\sim}{\mathbf{1}}, \underset{\sim}{\mathbf{1}}, 0), \quad \Phi_{W}=(\underset{\sim}{\mathbf{1}}, \underset{\sim}{\mathbf{3}}, 0), \quad \Phi_{G}=(\underset{\sim}{\mathbf{8}}, \underset{\sim}{\mathbf{1}}, 0) .
$$

Let us note that they form, together with the vector superfields $V_{B}, V_{W}$ and $V_{G}$, three complete vector representations of the $N=2$ supersymmetric algebra.

The Higgsino fields can be rendered massive by mixing the two Higgs chiral superfields $H_{D}$ and $H_{U}$ with their $R$-partners, the chiral superfields $R_{D}$ and $R_{U}$,

$$
R_{D}=\left(\underset{\sim}{\mathbf{1}}, \underset{\sim}{\mathbf{2}}, \frac{1}{2}\right), \quad R_{U}=\left(\underset{\sim}{\mathbf{1}}, \underset{\sim}{\mathbf{2}},-\frac{1}{2}\right) .
$$

The notations related to the component fields of these five new superfields are shown in Table 3 .

Kinetic and gauge interaction terms for the new superfields $\Phi_{B}, \Phi_{W}$ and $\Phi_{G}$ are given by the canonical Kähler potential of Eq. (10),

$$
\mathcal{L}_{K}=\int \mathrm{d}^{2} \theta \mathrm{d}^{2} \bar{\theta}\left[\Phi_{B}^{\dagger} \Phi_{B}+\Phi_{W}^{\dagger} e^{-2 g_{w} \tilde{V}_{W}} \Phi_{W}+\Phi_{G}^{\dagger} e^{-2 g_{s} \tilde{V}_{G}} \Phi_{G}\right]
$$

where $\tilde{V}_{W}=V_{W^{k}} T_{k}$ and $\tilde{V}_{G}=V_{G^{a}} T_{a}$. The vector superfields $V_{W^{k}}$ and $V_{G^{a}}$ have been defined in Eq. (11), and, in this case, the matrices $T_{k}$ and $T_{a}$ are taken as the representation matrices of the $S U(2)$ and $S U(3)$ algebra in the adjoint representation. The Lagrangian of Eq. (19) contains couplings of the new scalar adjoint 
Table 3. $R$-partners of the vector and Higgs superfields in the minimal $R$-symmetric supersymmetric model. They are given together with their scalar and fermionic components, as well as with their representations under the $S U(3)_{c} \times S U(2)_{L} \times U(1)_{Y}$ gauge group.

\begin{tabular}{|c||c|c|c|}
\hline Superfield & Fermion & Scalar & Representation \\
\hline \hline$\Phi_{B}$ & $\widetilde{B}^{\prime}$ & $\sigma_{B}$ & $(\mathbf{1}, \mathbf{1}, 0)$ \\
$\Phi_{Y}$ & $\widetilde{W}^{\prime}$ & $\sigma_{W}$ & $(\mathbf{1}, \mathbf{3}, 0)$ \\
$\Phi_{G}$ & $\widetilde{g}^{\prime}$ & $\sigma_{G}$ & $(\mathbf{8}, \mathbf{1}, 0)$ \\
\hline & \multirow{2}{*}{$\widetilde{R}_{u}=\left(\begin{array}{c}\widetilde{R}_{u}^{0} \\
\widetilde{R}_{u}^{-}\end{array}\right)$} & $R_{u}=\left(\begin{array}{c}R_{u}^{0} \\
R_{u}^{-}\end{array}\right)$ & $\left(\mathbf{1}, \mathbf{2},-\frac{1}{2}\right)$ \\
& & & \\
& & & \\
$R_{U}$ & $\widetilde{R}_{d}=\left(\begin{array}{c}\widetilde{R}_{d}^{+} \\
\widetilde{R}_{d}^{0}\end{array}\right)$ & $R_{d}=\left(\begin{array}{c}R_{d}^{+} \\
R_{d}^{0}\end{array}\right)$ & $\left(\mathbf{1}, \mathbf{2}, \frac{1}{2}\right)$ \\
& & & \\
\hline
\end{tabular}

$\sigma$-fields to a single gauge boson and to pairs of gauge bosons through the usual covariant derivatives. The gauge-invariant kinetic terms of the $R$-superfields are given as in the second term of Eq. (14), the representation matrices of $S U(2)$ being taken in the fundamental representation.

Interactions among the chiral superfields of the theory is given by the superpotential

$$
\begin{aligned}
W(\Phi)= & \left(\mathbf{y}^{\mathbf{u}}\right)_{i j} U_{R}^{i} Q_{L}^{j} \cdot H_{U}-\left(\mathbf{y}^{\mathbf{d}}\right)_{i j} D_{R}^{i} Q_{L}^{j} \cdot H_{D}-\left(\mathbf{y}^{\mathbf{e}}\right)_{i j} E_{R}^{i} L_{L}^{j} \cdot H_{D} \\
& +\sum_{i=U, D}\left[\lambda_{i}^{B} H_{i} \Phi_{B} Y R_{i}+\lambda_{i}^{W} H_{i} \Phi_{W^{k}} \frac{\sigma_{k}}{2} R_{i}+\mu_{i} H_{i} \cdot R_{i}\right] .
\end{aligned}
$$

In the expression above, $\mathbf{y}^{\mathbf{u}}, \mathbf{y}^{\mathbf{d}}$ and $\mathbf{y}^{\mathbf{l}}$ denote the $3 \times 3$ Yukawa matrices in generation space and these terms are also present in the MSSM. In contrast, the other terms are related to the $R$-partner superfields. The parameters $\mu_{i}$ are the $(R$-)Higgs off-diagonal mass-mixing parameters and the dot products again stand for $S U(2)$ invariant products. The $\lambda$-couplings are the trilinear couplings of the $R$-partners of the $U(1)_{Y}$ and $S U(2)_{L}$ vector superfields with the $(R$-)Higgs superfields.

Soft-supersymmetry breaking originates from hidden sector spurions which preserve the $R$-symmetry. In the most general version of the model, both $F$-type and $D$-type supersymmetry breaking terms are allowed. In the first case, a spurion chiral superfield $X$ gets a vacuum expectation value $\langle X\rangle=\theta \cdot \theta v_{F}$ whilst in the second case, a spinorial field strength tensor $W_{\alpha}^{\prime}$ gets a vacuum expectation value $\left\langle W_{\alpha}^{\prime}\right\rangle=\sqrt{2} \theta_{\alpha} v_{D}$, the quantities $v_{F}$ and $v_{D}$ being related to the order of magnitude $M_{\mathrm{SUSY}}$ of the supersymmetric masses.

Dirac gaugino masses arise through dimension-five operator generating mixing between the fermionic component of the chiral superfield $\Phi_{k}$ and the gaugino com- 
ponent of the vector superfield $V_{k}$, with $k=B, W, G$,

$$
\mathcal{L}_{\text {soft }, 1}=\sum_{k=B, W, G} \frac{1}{2 g_{k}} m_{1}^{k} \int \mathrm{d}^{2} \theta \frac{W^{\prime \alpha}}{M_{\mathrm{SUSY}}} W_{k \alpha} \Phi_{k}
$$

This involves a $D$-type spurion, $W_{k}$ being the superfields strength tensor associated to the vector superfield $V_{k}$ and $m_{1}^{k}$ the corresponding mass parameter. The overall normalization factor $1 /\left(2 g_{k}\right)$ depending on the gauge coupling constant ensures a correct normalization of the Dirac mass term.

The $F$-type spurion allows to write down mass terms for the scalar sector of the theory,

$$
\mathcal{L}_{\text {soft }, 2}=\sum_{\Phi=\text { chiral superfields }} \int \mathrm{d}^{2} \theta \mathrm{d}^{2} \bar{\theta} m_{\Phi}^{2} \frac{X X^{\dagger}}{M_{\text {SUSY }}^{2}} \Phi^{\dagger} \Phi
$$

The sum runs over the chiral superfields included in both Table 2 and Table 3 However, both $F$-type and $D$-type spurions allow to generate additional mass terms for the scalar adjoint $\sigma$-fields through the dimension-six operators

$$
\mathcal{L}_{\text {soft }, 3}=\sum_{k=B, W, G} \int \mathrm{d}^{2} \theta \mathrm{d}^{2} \bar{\theta}\left[\left(m_{2}^{k}\right)^{2} \frac{X X^{\dagger}}{M_{\mathrm{SUSY}}^{2}}+\left(m_{3}^{k}\right)^{2} \frac{W^{\prime} \cdot W^{\prime}}{M_{\mathrm{SUSY}}^{2}}\right] \operatorname{Tr}\left[\Phi_{k} \Phi_{k}\right] .
$$

Assuming real soft-supersymmetry breaking masses, the two real degrees of freedom included in each of the complex $\sigma$-scalar fields do not mix. Consequently, we have purely scalar or purely pseudoscalar mass-eigenstates. For the sake of simplicity, this hypothesis is adopted in this work and the pseudoscalar state are neglected.

Finally, the soft-supersymmetry breaking Lagrangian also contains bilinear $(R-)$ Higgs interactions driven by the $F$-type spurion,

$$
\mathcal{L}_{\text {soft }, 4}=\int \mathrm{d}^{2} \theta \mathrm{d}^{2} \bar{\theta} \frac{X X^{\dagger}}{M_{\text {SUSY }}^{2}}\left[B_{U} H_{U} \cdot R_{U}+B_{D} H_{D} \cdot R_{D}+B H_{u} \cdot H_{D}\right],
$$

where $B, B_{U}$ and $B_{D}$ are the corresponding mixing strengths.

More importantly for hadron-collider phenomenology, the new scalar fields also couple singly to a pair of quarks and to a pair of gluons through loop-diagrams involving squarks, gluinos, neutralinos and charginos. Enhanced by the relative magnitude (at the weak scale) of the strong coupling, we only keep the dominant interactions involving a sgluon field $\sigma_{G}$. They can be described by the effective Lagrangian

$$
\begin{aligned}
\mathcal{L}_{\mathrm{eff}}= & \sigma_{G}^{a}\left[\left(\lambda_{L}\right)^{f}{ }_{f^{\prime}} \bar{\Psi}_{q f} P_{L} T_{a} \Psi_{q}^{f^{\prime}}+\left(\lambda_{R}\right)^{f}{ }_{f^{\prime}} \bar{\Psi}_{q f} P_{R} T_{a} \Psi_{q}^{f^{\prime}}\right] \\
& +\lambda_{g} d^{a b c} \sigma_{G a} G_{\mu \nu b} G^{\mu \nu}{ }_{c}+\text { h.c. } .
\end{aligned}
$$

In this last equation, we have introduced $\Psi_{q}^{f}$ as the Dirac field associated to a quark of flavor $f, G_{\mu \nu b}$ as the gluon field strength tensor and $d^{a b c}$ as the $S U(3)$ symmetric tensor. The strengths of the interactions included in the effective Lagrangian $\mathcal{L}_{\text {eff }}$, i.e., the parameters $\lambda_{L}, \lambda_{R}$ and $\lambda_{g}$, are taken as free parameters even if in principle, they fully depend on the particle spectrum and sfermion mixings. 


\subsubsection{Implementation in FEYNRules}

As in Section 2.2, the implementation in FeynRules of the model described above can be performed in a very efficient way starting from the existing implementation of the MSSM. However, unlike the $R$-parity violating model, all the novelties cannot be included in an additional file only. The declaration of the gluino and neutralino fields must indeed be modified, since they are now Dirac instead of Majorana fermions, and this requires a modification of the MSSM implementation. Therefore, we start from a copy of the file mssm. fr and include all the modifications presented in Section 2.3 .1 .

The implementation of the five new chiral superfields as well as the one of all the model free parameters strictly follow the rules presented in Ref. 22 and Ref. 25. Whilst the implementation of the parameters and the one of the $R$-Higgs superfields is immediate, let us take the example of the $\Phi_{G}$ superfield to briefly comment on the implementation of the $R$-partners of the vector superfields of the MSSM. A possible set of Mathematica commands for the declaration of the superfield $\Phi_{G}$ reads

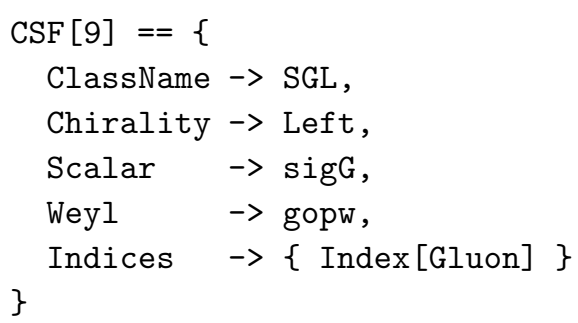

where we have assigned the symbols sigG and gopw to the sgluon $\sigma_{G}$ and the gluino $\tilde{g}^{\prime}$ field, respectively. For the implementation of the component fields, we have,

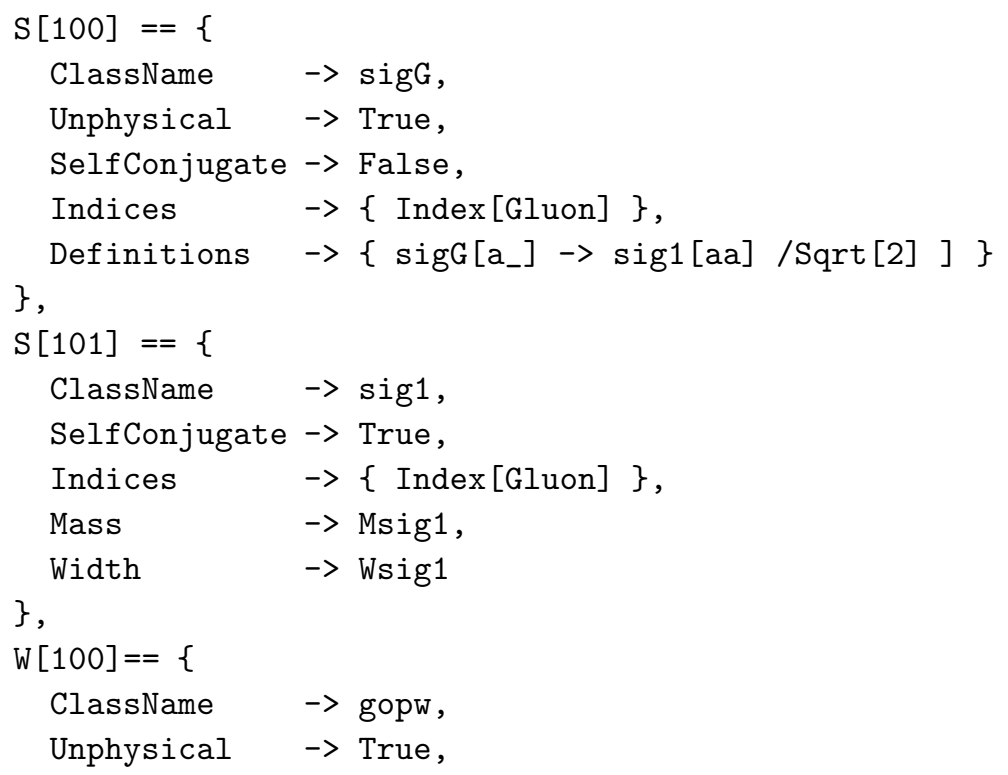




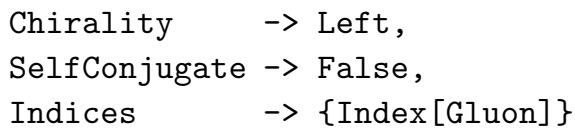

Before moving on, let us comment on the way used to implement the mixing of the real degrees of freedom included in the complex sgluon field. Whilst the symbol sigG represents the complex scalar field which is the unphysical component field of the chiral superfield $\Phi_{G}$, the symbol sig1 is the real scalar (and not pseudoscalar) degree of freedom included in $\sigma_{G}$. The pseudoscalar state has indeed been decoupled and removed from the mixing relation implemented in the option Definitions of the class sigG. The $\widetilde{g}^{\prime}$ field has been associated to the symbol gopw. Denoting by gow the gluino $\widetilde{g}$ two-component fermion embedded in $V_{G}$, the two fields $\widetilde{g}$ and $\widetilde{g}^{\prime}$ can be related to the (Dirac) four-component gluino field through the option

WeylComponents -> \{gow, gpowbar\}

of the gluino instance of the particle class.

We now turn to the $R$-symmetric Lagrangian presented in Section 2.3.1 All the gauge-invariant kinetic terms which are given in Eq. (14) and Eq. (19) are automatically computed by issuing the commands

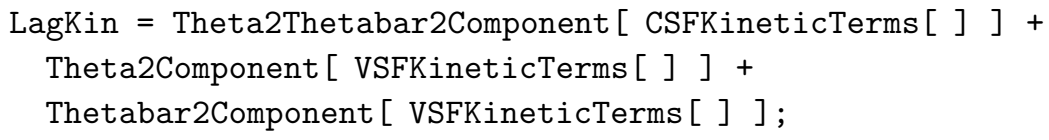

The superpotential can also be immediately implemented from Eq. (20), translating the textbook expression into a MAтнематісA declaration of a variable Superw,

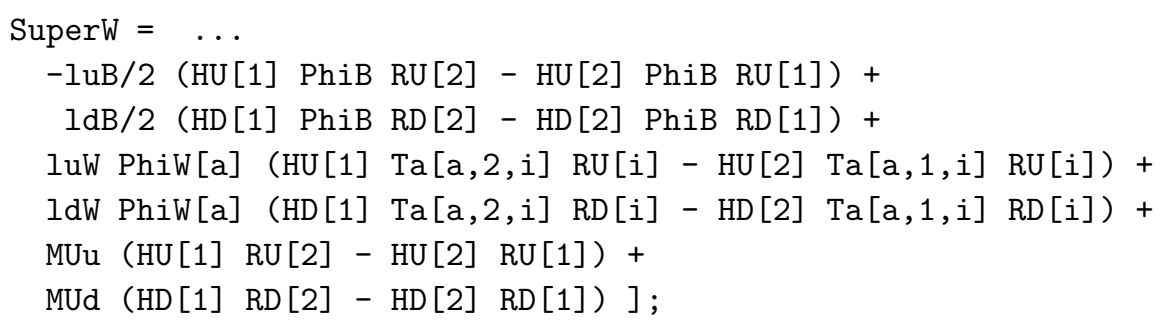

where the dots stand for the trilinear Yukawa interactions identical as in the MSSM and then omitted for brevity 25 . In the implementation above, the $S U(2)_{L}$ contractions have been expanded, $\mathrm{Ta}$ are the symbols representing the fundamental representation matrices of $S U(2)_{L}$, HU, HD, RU and RD are the names of the classes associated to the $\left(R\right.$-)Higgs superfields, and PhiB and PhiW to the superfields $\Phi_{B}$ and $\Phi_{W}$. The superpotential $\lambda$-parameters of the superpotential are denoted by luB, $1 \mathrm{~dB}, 1 \mathrm{uW}$ and $\mathrm{ldW}$ whilst the $\mu$-parameters are given as MUu and MUd. The associated Lagrangian can then be computed as

$$
\text { Lag }=\text { Theta2Component }[\text { SuperW }]+\operatorname{Thetabar2Component~}[\text { HC }[\text { SuperW }]] \text {; }
$$


Finally, the soft-supersymmetry breaking Lagrangian split between Eq. (21), Eq. (22), Eq. (23) and Eq. (24) can also be implemented directly within the superspace module of FeynRules. As an example, we take the gluino Dirac mass term implemented in the variable mglno which could be included in the model file as

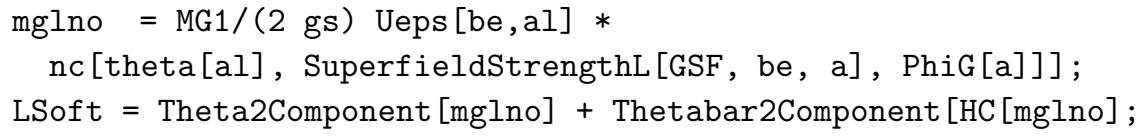

where MG1 is the symbol associated to the product of the soft supersymmetrybreaking mass by the vacuum expectation value of the spurion superfield strength tensor $W^{\prime}$, i.e., $m_{1}^{G} v_{D}$. For the conventions on the rank-two fully antisymmetric tensor UEps and on the nc environment, we refer to Ref. 25.

\section{From FeynRules to MadGraph}

\subsection{General features}

Among the whole set of existing automated Monte Carlo tools allowing to address phenomenological studies of high-energy physics processes, most all of them contain restrictions on the color and/or Lorentz structures which can appear in the interaction vertices of any model. While all the structures included in the Standard Model and the MSSM are generally allowed, vertices with non-standard color and/or Lorentz structures are most of the time not supported and must be discarded from the model implementations. As a consequence, this constrains beyond the Standard Model phenomenological explorations which could be performed with the help of an automated Monte Carlo tool. One is thus forced to compute squared matrix element in a non-automated fashion, e.g., by hand, and implement the results into non-automated tools such as Herwig 6869 or PYTHIA 70/71. Going beyond two-to-two scattering processes is thus a rather tedious task.

In this work, we present a way to overcome those limitations with the use of the MAdGraph 5 matrix element generator 15 and the UFO model format 32 . We focus on the non-trivial color structures appearing in the Lagrangians of the theories presented in Section 2, namely the rank-three fully antisymmetric tensor in color space $\epsilon_{\ell m n}$, where $\ell, m$ and $n$ are (anti)fundamental color indices, and the symmetric tensor of $S U(3), d^{a b c}$, where $a, b$ and $c$ are adjoint color indices.

The Monte Carlo event generator MADGRAPH 5 allows for the automated generation of tree-level matrix elements associated to any scattering process in a very efficient way. In particular, MADGRAPH 5 is thus a suitable tool to simulate final state signatures such as those produced in the high energy proton-proton collisions occurring at the LHC. The task of the user consists mainly in specifying the process of interest in terms of initial and final state particles, the collision basic information (such as the energy of the colliding beams), a set of basic cuts related to the analysis aimed to be performed and, of course, the particle physics model under consideration, renormalizable or not. 
The model library of MAdGraph 5 is built upon the FeynRules model database. Information is passed from FeynRules to MADGRAPH through the universal output format of FEYNRULES, dubbed the UFO. This format has been designed to overcome the above-mentioned restrictions on the allowed Lorentz and color structures for the interaction vertices at the level of the Monte Carlo tools. The key features are flexibility and modularity through the use of PYтHON classes and objects to represent particles, parameters and vertices, saving thus the model information in an abstract fashion, also independent from any Monte Carlo program. Consequently, it is universal in the sense that it is not tied to any specific matrix element generator. Presently, two generators, MADGRAPH and GoSAM, are currently using it as their standard model format, and the future version of HERWIG ++ will also be based on the UFO format.

In order to translate a FEYNRULES model implementation, such as one of those described in Section 2 an automated interface has been included within the public version of the FEYNRULES program. It can be called by typing, in a MATHEMATicA session, the command

\section{WriteUFO[ Lag ] ;}

where the variable Lag contains the Lagrangian of the considered model, expressed in terms of the usual scalar fields, (four-component) fermions, gauge bosons (together with the corresponding ghost fields, if relevant) and tensorial fields of particle physics. Let us note that all the states are assumed to be rotated to the mass eigenbasis. Once issued, the WriteUFO function internally calls the FeYnRules core function FeynmanRules in order to compute all the vertices associated to the Lagrangian Lag. They are then expanded into a color $\otimes$ spin basis,

$$
\mathcal{V}^{a_{1} \ldots a_{n}, \ell_{1} \ldots \ell_{n}}\left(p_{1}, \ldots, p_{n}\right)=\sum_{i, j} C_{i}^{a_{1} \ldots a_{n}} G_{i j} L_{j}^{\ell_{1} \ldots \ell_{n}}\left(p_{1}, \ldots, p_{n}\right)
$$

where the variables $p_{i}$ denote the particle momenta and $G_{i j}$ the coupling strengths. The quantities $C_{i}^{a_{1} \ldots a_{n}}$ and $L_{j}^{\ell_{1} \ldots \ell_{n}}\left(p_{1}, \ldots, p_{n}\right)$ are tensors in color and spin space, respectively. These tensors act as a basis in the color $\otimes$ spin space and could be used by several vertices, reducing by this way possible redundancies in a model implementation. The coupling strengths of a specific vertex are therefore the coordinates of the vertex in this basis. Following this structure, vertices are implemented in the UFO format by using several different PYTHON objects and their attributes to represent the vertices themselves, their Lorentz and color structures and the coupling strengths.

Once all the Lagrangian vertices are decomposed as in Eq. (26), the UFO interface writes a set of PYTHON files stored in a single directory. These files contain all the model information, from the definition of the particle content and the parameters as instances of the generic UFO particle and parameter class, respectively, to the implementation of the (factorized) vertices as instances of the vertex class.

In order to use the produced UFO model with MADGRAPH, the outputted files 
must be copied into the models directory of MADGRAPH so that they can be used for event generation as any other built-in model.

As soon as a process is specified by the user, MADGRAPH 5 internally calls the AlOHA package 34 which generates the subroutines, based on the HelAs library $35|36| 37 \mid 38$, necessary to compute the helicity amplitudes related to the process under consideration from the UFO. This allows for an efficient evaluation of the associated squared matrix element. Indeed, helicity amplitudes include helicity wavefunctions corresponding to specific substructures included in a given Feynman diagram, which can be further reused within different diagrams.

Supersymmetric theories contain, in their most general form, more than several thousands of vertices. A large class of vertices are however vanishing in scenarios of phenomenological interests at colliders. As an example, let us take the sector of the up-type squarks (the six scalar partners of the three generations of left-handed and right-handed up-type quarks). Since after electroweak symmetry breaking, all particles with the same spin, color representation and electric charge mix, the six up-type squark gauge-eigenstates undergo a $6 \times 6$ mixing. Consequently, the scalar potential contains $\mathcal{O}(1000)$ four scalar interactions among the up-type squark mass-eigenstates which violate flavor most of the time. However, the flavorviolating entries of the associated mixing matrix are, in most phenomenologically viable benchmark scenarios, vanishing, which renders a large part of the $\mathcal{O}(1000)$ interaction vertices equal to zero and irrelevant.

The presence of zero vertices in UFO model files, or more generally into model files for any matrix element generator, considerably slows down event generation, since they must be loaded in the computer memory on run time and diagrams with a vanishing contribution are effectively generated. Therefore, it may be suitable to remove these vertices from the UFO model files to speed up the evaluation of the matrix elements, making it much more efficient. This task can be done at the FEYNRULEs-level with the help of the WriteRestrictionFile and LoadRestriction commands.

Firstly, the numerical values of all the model parameters, i.e., a full supersymmetric spectrum, must be loaded in FEYnRules. Since the instances of the parameter class included in FEYNRULES models are organized following a structure inspired by the Supersymmetry Les Houches Accord (SLHA), the numerical initialization of the model parameters can be achieved by loading a SLHA file directly into the Mathematica session,

$$
\text { ReadLHAFile[Input } \rightarrow \text { "susy.dat"]; }
$$

where susy. dat is the filename of the spectrum provided by, e.g., one of the dedicated existing supersymmetric spectrum generators. The detection of the vanishing parameters is performed by issuing the following sequence of MATHEMATICA commands,

WriteRestrictionFile [ ] ; 


\section{LoadRestriction ["ZeroValues.rst"] ;}

The WriteRestrictionFile function asks FeynRules to scan over the whole set of internal and external parameters and write, in a file named ZeroValues.rst, a list of MatнематісA replacement rules mapping all the vanishing parameters to zero. The LoadRestriction function allows to read this file and load the list of rules into FEYNRULES so that it could be used, at a later stage, by either the UFO interface or by any other FEYNRules interface to a Monte Carlo program. When called, the interfaces apply this set of replacement rules to each vertex derived by the function FeynmanRules before translating it to be written to the output files. The zero contributions are hence immediately simplified and dropped. If after the mapping, a full vertex is numerically evaluated to zero, it is ignored and not written at all in the generated output files.

After this optimization, it can be noted that among all the remaining interactions, hundreds of vertices consist in four scalar interactions which are, for tree-level computations, in general phenomenologically less relevant. Therefore, again for the sake of efficiency at the Monte Carlo tool level, it is useful not to include those vertices. This task can be done through the Exclude4Scalars option of the FEYNRules interfaces. In the UFO case, one would have to issue the Mathematica command

WriteUFO[ Lag, Exclude4Scalars -> True ];

Let us note that one must keep in mind that the UFO model files generated in the optimized way illustrated above are not fully general and highly dependent on the considered benchmark scenario (defined here in the file susy.dat), even if at the FeynRules level, the model implementation is as general as possible.

\section{Benchmark scenarios and processes of interest}

\subsection{The R-parity violating Minimal Supersymmetric Standard Model}

The ATLAS and CMS experiments are currently setting impressive limits on the masses of the supersymmetric particles and excluding a significant part of the supersymmetric parameter space $3 \mid 4$. In order to reinterpret the experimental results in terms of as many different manifestations of supersymmetry as possible, specific benchmark scenarios have been recently proposed 72 . Even if a large emphasis is put on the constrained version of the MSSM, the $R$-parity violating case is also addressed. We therefore adopt as a benchmark scenario for our $R$-parity violating supersymmetric exploration one of the proposed points, along a line in the $R$-parity violating MSSM parameter space dubbed the 'RPV3-line 72 .

This line is inspired by the constrained MSSM. Each benchmark lying on it is thus defined by four free parameters defined at the grand unification scale and the sign of the bilinear Higgs mixing superpotential $\mu$-parameter. To this restricted set 
of supersymmetric input parameters, one must supplement the value of one of the $\lambda^{\prime \prime}$ coupling, assuming thus the single coupling dominance hypothesis $\underline{6162}$ and neglecting any other $\lambda^{\prime \prime}$, together with all the $\lambda, \lambda^{\prime}, T, T^{\prime}$ and $T^{\prime \prime}$ parameters. This line is thus perfectly suitable to probe the exotic color structures included in the superpotential part of the Lagrangian.

We fix the universal scalar mass $m_{0}=100 \mathrm{GeV}$, the universal gaugino mass $m_{1 / 2}=400 \mathrm{GeV}$ and the universal trilinear coupling $A_{0}=0 \mathrm{GeV}$. The ratio of the vacuum expectation values of the neutral component of the two Higgs doublets is taken as $\tan \beta=10$, whilst the $\mu$-parameter is chosen positive.

For the Standard Model sector, we fix the top quark pole mass to $m_{t}=173.2$ $\mathrm{GeV} 73$, the bottom quark mass to $m_{b}\left(m_{b}\right)=4.2 \mathrm{GeV}$ and the $Z$-boson mass to $m_{Z}=91.1876 \mathrm{GeV}$. The Fermi constant has been taken as $G_{F}=1.16637 \times 10^{-5}$ $\mathrm{GeV}^{-2}$, and the strong and electromagnetic coupling constants at the $Z$-pole as $\alpha_{s}\left(m_{Z}\right)=0.1176$ and $\alpha\left(m_{Z}\right)^{-1}=127.934$, according to the Particle Data Group Review 74 .

There exists a wide range of experimental measurements constraining the magnitude of the baryon-number violating $\lambda^{\prime \prime}$ parameters, such as data related to, e.g., $K-\bar{K}$ systems $\frac{75 \mid 76 / 77}{}$, neutron electric dipole moments ${ }^{75}$, rare hadronic $B$-decays 78/79, nucleon-antinucleon oscillations, as well as to double $80|81| 82$ and single nucleon decays 838485 . However, the most restrictive bounds on the $\lambda^{\prime \prime}$ parameters are deduced from cosmological and astrophysical data with the observed flux of antiproton cosmic rays 86 . All these limits are nevertheless not applicable on the $\lambda^{\prime \prime}{ }_{3 j k}$ couplings, related to the top (s)quarks, for supersymmetric scenarios where the lightest supersymmetric particle is lighter than the top quark. In this case, the $\lambda^{\prime \prime}{ }_{3 j k}$ parameters are left almost unconstrained, which is particularly appealing for LHC physics. We choose $\lambda_{312}=0.2$, since with our setup, at it is presented below, the lightest supersymmetric particle is lighter than the top quark.

The input parameters for the chosen benchmark point are summarized in Table 4. where we remind that the supersymmetric parameters are defined at the grand unification scale.

Table 4. Input parameters associated to the chosen benchmark scenario in the context of the $R$-parity violating MSSM. All other $\lambda^{\prime \prime}$-parameters, together with the $\lambda$, $\lambda^{\prime}, T, T^{\prime}$ and $T^{\prime \prime}$ parameters are taken equal to zero.

\begin{tabular}{|cccccc|}
\hline$m_{t}[\mathrm{GeV}]$ & $m_{b}[\mathrm{GeV}]$ & $m_{Z}[\mathrm{GeV}]$ & $G_{F}\left[\mathrm{GeV}^{-2}\right]$ & $\alpha_{s}\left(m_{Z}\right)$ & $\alpha\left(m_{Z}\right)^{-1}$ \\
\hline 173.2 & 4.2 & 91.1876 & $1.16637 \times 10^{-5}$ & 0.1176 & 127.934 \\
\hline \hline$m_{0}[\mathrm{GeV}]$ & $m_{1 / 2}[\mathrm{GeV}]$ & $A_{0}[\mathrm{GeV}]$ & $\tan \beta$ & $\operatorname{sign}(\mu)$ & $\lambda_{312}^{\prime \prime}$ \\
\hline 100 & 400 & 0 & 10 & $>0$ & 0.2 \\
\hline
\end{tabular}

The soft supersymmetry-breaking masses and interaction strengths at the electroweak scale are obtained through renormalization group running using the SPHENo 3 package [87/88, which solves the renormalization group equations nu- 

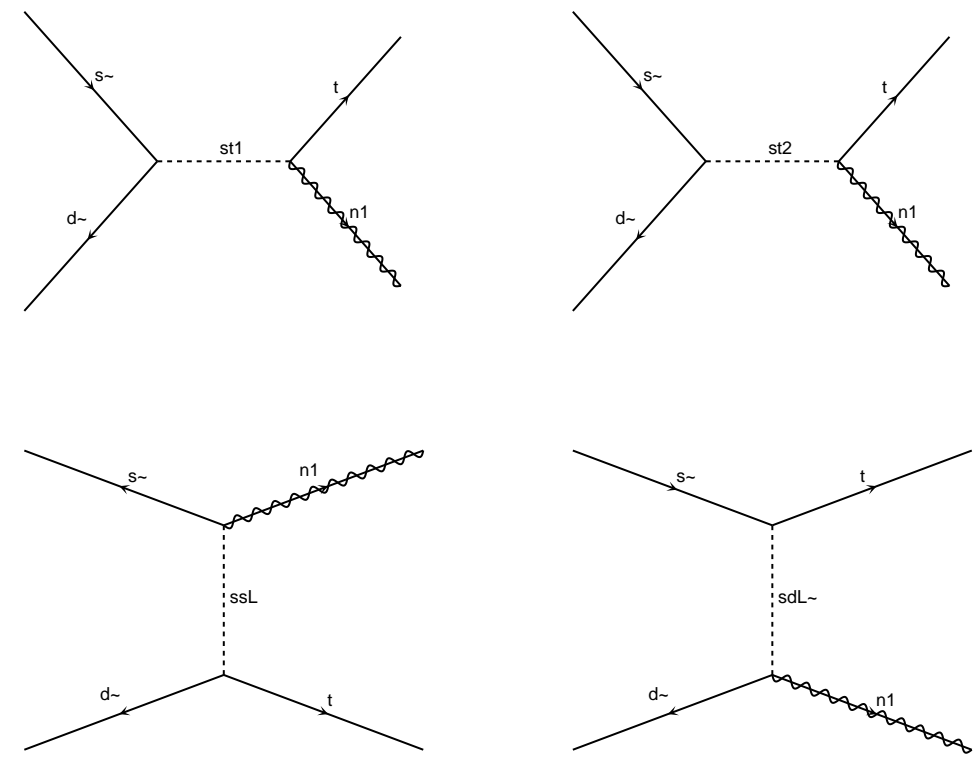

Fig. 1. Feynman diagrams associated to $R$-parity violating monotop production as generated by MADGRAPH 5. In the notations above, the label $\mathrm{n} 1$ stands for the lightest neutralino, $\mathrm{s} \sim$ and $\mathrm{d} \sim$ for strange and down antiquarks and $t$ for a top quark. Attached to the internal lines, st1 and st2 represent the two top squarks, ssL the left-handed strange squark and sdL $\sim$ the left-handed down antisquark.

merically to two-loop order. This program then extracts the particle spectrum and mixings at the two-loop level for the Higgs sector and at the one-loop level for all the other particles.

In the sector of the electroweak superpartners, the sleptons are fairly light, with masses of $\mathcal{O}(200-300) \mathrm{GeV}$, and the neutralino and chargino masses range from $160 \mathrm{GeV}$ for the lightest neutralino, being the lightest supersymmetric particle, to $550 \mathrm{GeV}$ for the heavier states. Let us emphasize that since the lightest neutralino is lighter than the top quark, the conditions to evade the experimental bounds on the $\lambda^{\prime \prime}$ parameters are fulfilled. The choice of $\lambda^{\prime \prime}{ }_{312}=0.2$ is thus justified. In contrast, in the strong sector, the squark masses are a larger, even if still rather modest, ranging from $650 \mathrm{GeV}$ to $875 \mathrm{GeV}$, whilst the gluino is heavier than all the squarks with a mass of $900 \mathrm{GeV}$.

One can observe that in the scenario depicted above, top squarks and antisquarks can be singly produced at the LHC with a sensible rate from an initial associated pair of down and strange (anti)quarks. Employing the CTEQ6L1 set of parton densities 89 and for a center-of-mass energy of $7 \mathrm{TeV}$, the corresponding total 
hadronic cross section indeed reach the level of about $2 \mathrm{pb}$. Whereas top squarks dominantly decay to dijets, their observation in this channel is made difficult by the huge QCD background. In contrast, the produced (anti)squark can decay to an associated pair of a top quark and a lightest neutralino with a branching ratio of $15 \%$.

As $R$-parity is violated through baryon-number violating operators, one can expect a multijet signature after the decay of both the top quark and the neutralino. However, in the considered scenario, the only possible three-body decay of the lightest neutralino, via a $\lambda^{\prime \prime}{ }_{3 j k}$ interaction, is not kinematically allowed and the only remaining option goes through a four-body decay. As a consequence, the lightest neutralino has a long lifetime and decays far outside the detector 90 . Therefore, the missing energy signature, typical from $R$-parity conserving supersymmetric scenarios, is still a convenient observable. This will be investigated in Section 5 where we carefully study the $R$-parity violating production of a single top quark in associated with missing energy, this signature being dubbed as a monotop signature 91. The corresponding Feynman diagrams, as generated by MADGRAPH 5, are presented in Figure 1.

\subsection{The Minimal R-symmetric supersymmetric model}

As stated above, most of the results of the ATLAS and CMS experiments are derived under the hypothesis of the constrained MSSM. Several reinterpretations exist in the context of popular models such as, e.g., the MSSM where supersymmetry is broken through gauge interactions, the so-called phenomenological MSSM or the Next-to-Minimal Supersymmetric Standard Model. However, there are alternative realizations of supersymmetry with highly different properties. Therefore, their investigation deserves dedicated studies in order to prepare the reinterpretation of the data. The minimal $R$-symmetric supersymmetric model is one of such examples, since it predicts, among others, the existence of a new color-octet scalar field (dubbed the sgluon) which is not present in more conventional experimentally covered supersymmetric theories. In this Section, we address the phenomenology of such as field, from its production at the LHC to its decay and signature within a detector.

In the model presented in Section 2.3, sgluons can be either singly or pairproduced in hadron collisions at high energies from initial states of quarks and gluons. Since the sgluon field belongs to the Standard Model sector of supersymmetric theoriesf, it is expected to decay mainly into light jets and/or top quarks, if kinematically allowed. Let us note even if squarks and gluinos could be lighter than the sgluon, which implies that additional decay channels might become possible, this case is not considered in this work. Subsequently, the superpartners and their

${ }^{\mathrm{f}}$ The sgluon field carries a positive $R$-parity quantum number, contrary to its gluino $\tilde{g}^{\prime}$ superpartner which has a negative $R$-parity. 
interactions are irrelevant for the phenomenological studies to be performed, and we therefore conceive a benchmark scenario where they decouple.

In order to optimize the generation of the MADGRAPH model files, the masses of the superpartners are set to a very high scale such as, e.g., $1000 \mathrm{TeV}$, and all the mixing matrices related to sfermions, neutralinos and charginos are set to zero. This makes all the interaction vertices involving two superpartners or more to vanish, so that they are effectively removed from the generated UFO model files by the optimization procedure described in Section 3 . In addition to the Standard Model inputs, it is then enough to fix the sgluon related parameters, i.e., its mass $m_{\sigma}$ and its couplings to quarks and gluons $\lambda_{L}, \lambda_{R}$ and $\lambda_{g}$ defined in Eq. (25). Let us note that even if the sgluon mass $m_{\sigma}$ depends, strictly speaking, on several soft parameters, i.e., $m_{2}^{G}, m_{3}^{G}$ and $m_{\Phi_{G}}$, we follow the simplifying approach to regroup all their contributions into a single parameter $m_{\sigma}$.

To investigate sgluonic signatures at the LHC, we consider a scenario where the sgluon is assumed to have a mass of $500 \mathrm{GeV}$, a rather collider-friendly value leading to a largely visible pair-production cross section at $7 \mathrm{TeV}$. The sgluon mass is thus high enough so that the $t \bar{t}$ decay channel is open (assuming non-vanishing $\lambda_{L}$ and/or $\lambda_{R}$ couplings). The production rate of a four top signature is hence enhanced with respect to the Standard Model expectation of about $0.3 \mathrm{fb}$, and the observation of such a signal might be a hint of the existence of a sgluon field. We impose that the sgluon singly couples only to top quarks and to gluons with strengths given by $\left(\lambda_{L}\right)^{3}{ }_{3}=\left(\lambda_{R}\right)^{3}{ }_{3}=0.3$ and $\lambda_{g}=1.5 \times 10^{-4}$, all other effective couplings being assumed vanishing.

The numerical values of the free parameters defining our simplified scenario are summarized in Table 5, together with the relevant Standard Model inputs. In this scenario, the sgluon-pair production cross section reach the level of $0.20 \mathrm{pb}$, whilst the sgluon-pair induced four top production cross section is of about $42 \mathrm{fb}$, i.e., more than about 140 times the Standard Model predictions. The corresponding Feynman diagrams, as generated by MADGRAPH 5, are presented in Figure 2 Whereas the value of the effective sgluon-gluon-gluon coupling of $1.5 \times 10^{-4}$ is rather reduced, it contributes significantly to sgluon-pair production and decays. Indeed, the first Feynman diagram of the second line of Fig. 2, involving two effective vertices, contributes to the sgluon-pair total production cross section by about $15 \%$. More importantly, the sgluon branching ratio to a gluon pair cannot be neglected, reaching more than $50 \%$, due to the phase-space suppression of the top-antitop pair channel.

Table 5. Input parameters associated to the chosen benchmark scenario in the context of investigating sgluon production in the minimal $R$-symmetric supersymmetric model. We remind that the superpartners are decoupled and thus irrelevant and that all the other $\lambda_{\{L, R\}}$ parameters are vanishing.

\begin{tabular}{|ccc|cccc|}
\hline$m_{t}[\mathrm{GeV}]$ & $m_{Z}[\mathrm{GeV}]$ & $\alpha_{s}\left(m_{Z}\right)$ & $m_{\sigma}[\mathrm{GeV}]$ & $\left(\lambda_{L}\right)^{3}{ }_{3}$ & $\left(\lambda_{R}\right)^{3}{ }_{3}$ & $\lambda_{g}$ \\
\hline 173.1 & 91.1876 & 0.1176 & 500 & 0.3 & 0.3 & 0.00015 \\
\hline
\end{tabular}



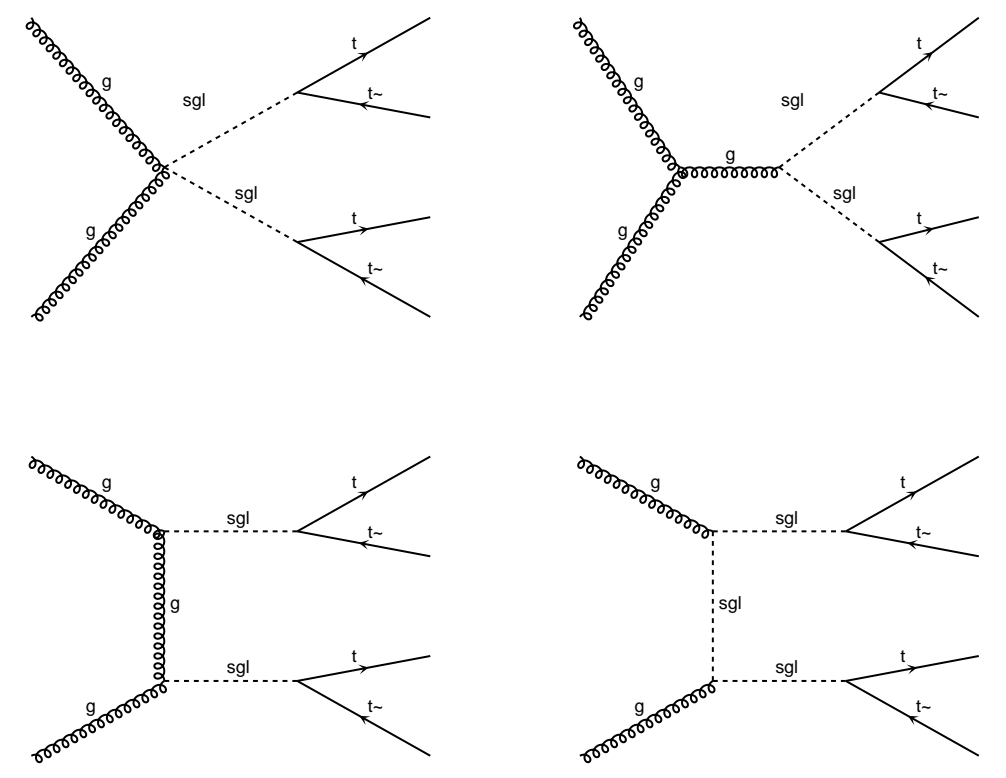

Fig. 2. Feynman diagrams associated to a four top signature issued from the production of a pair of sgluons, as generated by MADGRAPH 5 . In the notations above, the labels $t$ and $t \sim$ stand for the top quark and antiquark, $g$ for the gluon field and sgl for the sgluon. Let us note that $u$-channel diagrams are omitted since they can be deduced from the corresponding $t$-channel diagrams (lower panel).

\section{Non-minimal supersymmetric phenomenology}

Event simulation is performed for the LHC collider at a center-of-mass energy of $\sqrt{s}=7 \mathrm{TeV}$ and for an integrated luminosity of $4 \mathrm{fb}^{-1}$. Concerning both signal and background events, hard scattering matrix elements are described with the Monte Carlo generator MADGRAPH 515. Neglecting all quark masses but the top mass, we employ the leading order set of the CTEQ6 parton density fit 89 and identify both the renormalization and factorization scales as the transverse mass of the produced heavy particles. We then match the events generated by MADGraPH with parton showering and hadronization as provided by the PүтніA program. The version six of Pyтнia $[70$ is used for background and sgluon signal events, whilst the version ${ }_{8} 71$ is used for $R$-parity violating monotop signal events due to the exotic color structure not compliant with the requirements of PYTHIA 6. We finally perform a fast detector simulation with the program DeLPHES 92 , using the publicly available CMS detector card. Jets are reconstructed using the anti- $k_{t}$ algorithm with a radius parameter of $R=0.5$. The two examples of phenomenological analyses presented in this Section are performed with the help of the MADANALYsis 5 package 93 . 


\subsection{Monotop production in R-parity violating supersymmetry}

The main signatures associated with monotop production can be classified according to the top quark decay,

$$
p p \rightarrow t+\widetilde{\chi}_{1}^{0} \rightarrow b j j+\mathbb{E}_{T} \quad \text { or } \quad b \ell+\mathbb{E}_{T},
$$

where $j$ and $b$ denote light and $b$-jets, respectively and $\ell$ a charged lepton. The missing transverse energy $\mathbb{E}_{T}$ is associated to the lightest neutralino $\widetilde{\chi}_{1}^{0}$ escaping the detector invisibly due to its long lifetime 90 , as well as to the neutrino appearing in the case of leptonically decaying top quarks. Since leptonically decaying monotop signatures induced by $R$-parity violating supersymmetry have been widely investigated in the past 9495 , we therefore focus on monotop events where the top quark decays hadronically.

The only source of irreducible Standard Model background to an hadronic monotop signal consists in the production of an invisibly decaying $Z$-boson together with at least three jets, one of them being tagged as a $b$ jet. In contrast, there are many possible sources of (dominant) instrumental background related to detector effects. On the one hand, QCD multijet events with misreconstructed jets produce fake missing energy and mimic hence the monotop signature. However, asking for the reconstruction of a top quark could help to reject most of these QCD events. On the other hand, $W$ plus jets, $t \bar{t}$ and diboson events where the weak bosons decay to nonreconstructed leptons, as well as single top events including non-reconstructed or misrecontructed jets might also be a possible source of background.

In recent experimental analyses 96197 , it has been shown that simple selection cuts allow to keep a good control on the background. Inspired, in addition, by the parton-level results of Ref. 91, we start by requiring a large amount of missing transverse energy $\mathbb{E}_{T}>200 \mathrm{GeV}$. Within the employed simplified detector simulation, this selection cut allows to remove all the QCD multijet events from the background and to sensibly to reduce the contribution of the $t \bar{t}$ channel. We then impose a veto on the presence in the final state of any charged lepton (electron or muon) with a transverse momentum $p_{T}>10 \mathrm{GeV}$ and a pseudorapidity $|\eta|<2.5$. This last selection cut does not affect the signal but sensibly reduce the contributions of $Z$-boson plus jets and $W$-boson plus jets events.

In a second stage, we exploit the presence of a hadronically decaying top quark, together with the one of its decay products. We hence demand exactly one $b$-tagged jet with a transverse momentum $p_{T}>50 \mathrm{GeV}$ and a pseudorapidity $|\eta|<2.5$, as well as exactly two light jets with a transverse momentum $p_{T}>30 \mathrm{GeV}$ and a pseudorapidity $|\eta|<2.5$. We estimate a $b$-tagging efficiency depending on the transverse momentum of the jet as presented on Figure 3 (left panel) of Ref. 98, together with a charm and light jet mistagging rate (depending also on the fourmomentum of the jet) as on Figure 6 (right panel) of Ref. 99. Consequently, the efficiency of correctly tagging a jet with a transverse momentum of $50 \mathrm{GeV}$ as a $b$-jet is of about $70 \%$, whilst the mistagging rate of a charm (light) jet as a $b$-jet is of about $40 \%(2 \%)$. In addition, since the two light jets are issued from a $W$-boson, we 


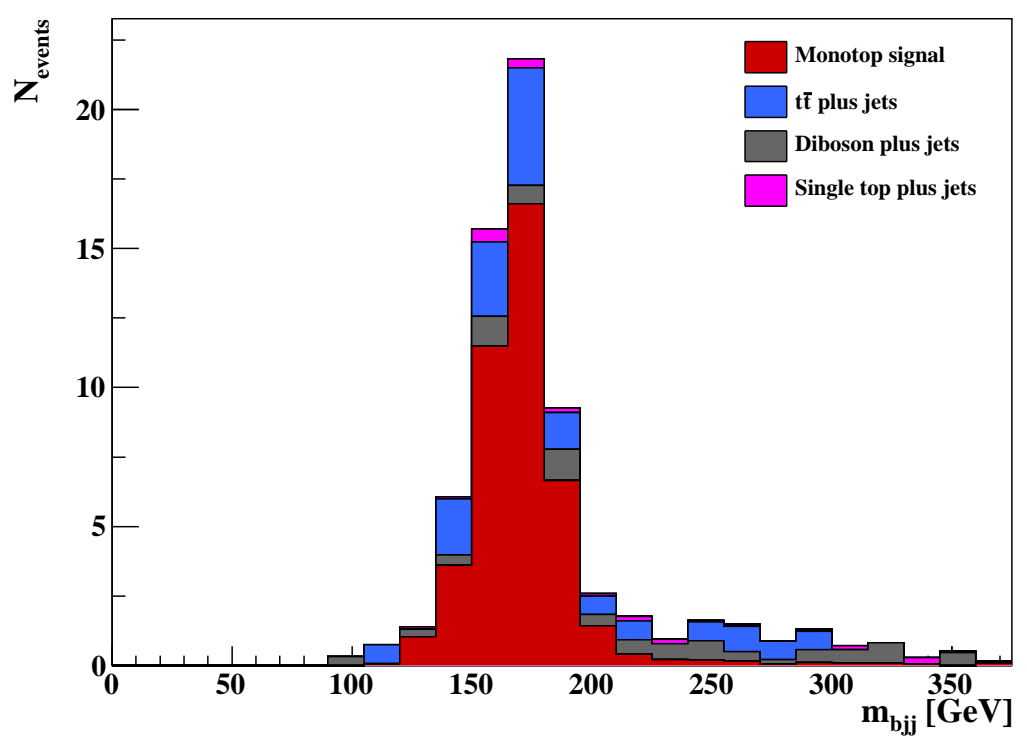

Fig. 3. We demand a monotop configuration of the final state as described in the text (large missing energy, no charged leptons, exactly one $b$-tagged jet and two light jets whose the invariantmass is compatible with a $W$-boson). After applying those selection cuts, we present the invariantmass distribution of the three jets $m_{b j j}$ both for the signal (red) and the dominant sources of background, i.e., single top (purple), $t \bar{t}$ (blue) and diboson (gray) events.

constrain their invariant-mass $m_{j j}$ to be compatible with the mass of the $W$-boson, i.e., $m_{j j} \in\left[m_{W}-15 \mathrm{GeV}, m_{W}+15 \mathrm{GeV}\right]$, with $m_{W}=80.31 \mathrm{GeV}$.

The distribution of the invariant-mass $m_{b j j}$ of the three jets is illustrated on Figure 3, After applying all the cuts described above, the dominant contributions to the Standard Model background are $t \bar{t}$ plus jets, diboson plus jets and single top plus jets events. In contrast, the contributions of all the other sources of background, such as $W$-boson, $Z$-boson or QCD events, are reduced to a barely visible level and thus under a very good control. We further constrain the system of the three selected jets by requiring their invariant-mass $m_{b j j}$ to be compatible with the mass of the top quark, lying thus in the range $\left[m_{t}-20 \mathrm{GeV}, m_{t}+20 \mathrm{GeV}\right]$. The final number of selected events is shown in Table 6 both for the signal and all the sources of background, together with the LHC sensitivity to a monotop signal. We define the latter as the number of signal events over the total number of (signal plus background) events $S / \sqrt{S+B}$, which reaches in our scenario the level of $4.95 \sigma$.

Conversely, a $3 \sigma$ deviation from the Standard Model expectation could be already observed for any value of the $R$-parity violating parameter $\lambda^{\prime \prime}{ }_{312}>0.11$, assuming the supersymmetric spectrum to be unchanged. Since the number of signal events is expected to be of the same order of magnitude for moderate superpartner 
Table 6. Number of remaining events $N_{\text {events }}$ for both the different sources of background and for the signal after the whole set of selection cuts described in the text. Contributions of the $W$-boson plus jets, the $Z$-boson plus jets and the QCD multijet channels are zero and thus not indicated. The results correspond to an integrated luminosity of $4 \mathrm{fb}^{-1}$ of proton-proton collisions at the LHC collider, running with a center-of-mass energy of 7 $\mathrm{TeV}$. The LHC sensitivity, defined as the number of signal events over the total number of (signal plus background) events $S / \sqrt{S+B}$ is also indicated.

\begin{tabular}{|c|c|}
\hline Event sample & $N_{\text {events }}$ after all the selection cuts presented in the text \\
\hline \hline Top-antitop pairs plus jets & $8.2 \pm 2.3$ \\
Diboson plus jets & $2.7 \pm 0.7$ \\
Single top & $0.9 \pm 0.3$ \\
\hline Total background & $11.8 \pm 2.4$ \\
Monotop signal & $33.2 \pm 1.0$ \\
Sensitivity & 4.95 \\
\hline
\end{tabular}

masses (below or around the $\mathrm{TeV}$ scale), the set of selection cuts presented above, i.e., standard monotop cuts, would be sufficient to render $R$-parity violating supersymmetric monotop signatures distinguishable for a large region of the constrained MSSM parameter space.

\subsection{Multitop production in minimal R-symmetric supersymmetry}

The top-enriched signature described in Section 4.2 leads to final states with a large multiplicity of jets and leptons as the decay products of the four top quarks. Therefore, the main sources of Standard Model background is expected to be related to rare processes such as the production of four top quarks or the production of a top-antitop pair in association with one or several gauge bosons.

In order to reject a good fraction of the Standard Model background events, we require the presence of exactly two charged leptons carrying the same electric charg£g, with a transverse momentum $p_{T}>20 \mathrm{GeV}$ and a pseudorapidity $|\eta|<2.5$. In addition, we require isolation criteria and reject leptons in the case they are at a relative distance $\Delta R<0.2$ of a jet.

Moreover, each of the leptons is issued from a leptonically decaying top quark. Therefore, it comes accompanied with a neutrino, carrying missing transverse energy, and we then ask for a missing transverse energy selection cut, keeping an event only if $\mathbb{E}_{T}>40 \mathrm{GeV}$.

As stated above, our signal events are rather rich in jets. In particular, we expect at least four $b$-tagged jets (one for each produced top quark) and four additional light jets issue from the hadronically decaying top quarks. Consequently, we only select events with at least eight jets, each of them having a transverse energy $E_{T}>20$ $\mathrm{GeV}$, and at least three of the jets are required to be $b$-tagged. As in Section 5.1 .

gIn our simplified detector simulation, the charge of a lepton is always correctly identified, which is far from being the case in experiments such as ATLAS or CMS. 


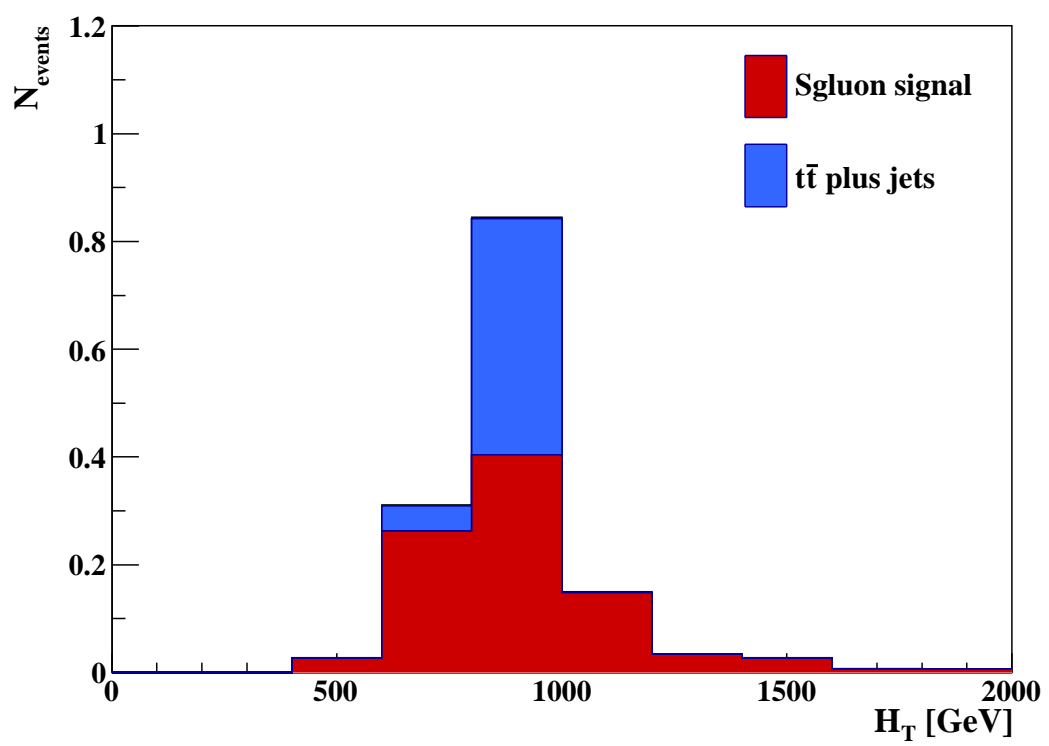

Fig. 4. Applying selection cuts related to a final state with an important hadronic activity (at least eight jets, three $b$-tagged jets) and containing two leptons with the same electric charge and a sensible missing energy, we present the distribution of the $H_{T}$ variable defined as the scalar sum of the transverse momentum of all the selected leptons and jets both for the signal (red) and the dominant source of background, i.e., $t \bar{t}$ (blue) events.

our $b$-tagging efficiency is estimated as on Figure 3 (left panel) of Ref. 98, together with a charm and light jet mistagging rate as on Figure 6 (right panel) of Ref. 99, We remind that all the efficiencies depend on the transverse momentum of the jet.

The important hadronic activity in the final state suggests to investigate the $H_{T}$ variable, defined as the scalar sum of the transverse momentum of all the selected leptons and jets. The results are presented in Figure 4. After applying the set of cuts described above, the dominant contributions to the background consist in $t \bar{t}$ events, as well as, in a smaller (and negligible) extent, in $t \bar{t}$ plus one or several gauge bosons events. This is also presented in Table 7 where we have omitted the non-contributing sources of background.

Our event selection criteria are very restrictive. However, due to the smallness of the signal cross section, these cuts are mandatory to ensure a good background rejection. In the case of higher luminosity or more favorable benchmark scenarios, one could however lower the requirements by demanding less jets with a transverse energy $E_{T}>20$, and/or a smaller number of $b$-tagged jets. The example of asking for six (seven) jets including three (two) $b$-tagged jets is shown in the upper (lower) panel of Figure 5. In this case, the background clearly dominates over the signal.

Even if the background rejection is pretty efficient with those cuts, a $4 \mathrm{fb}^{-1}$ 

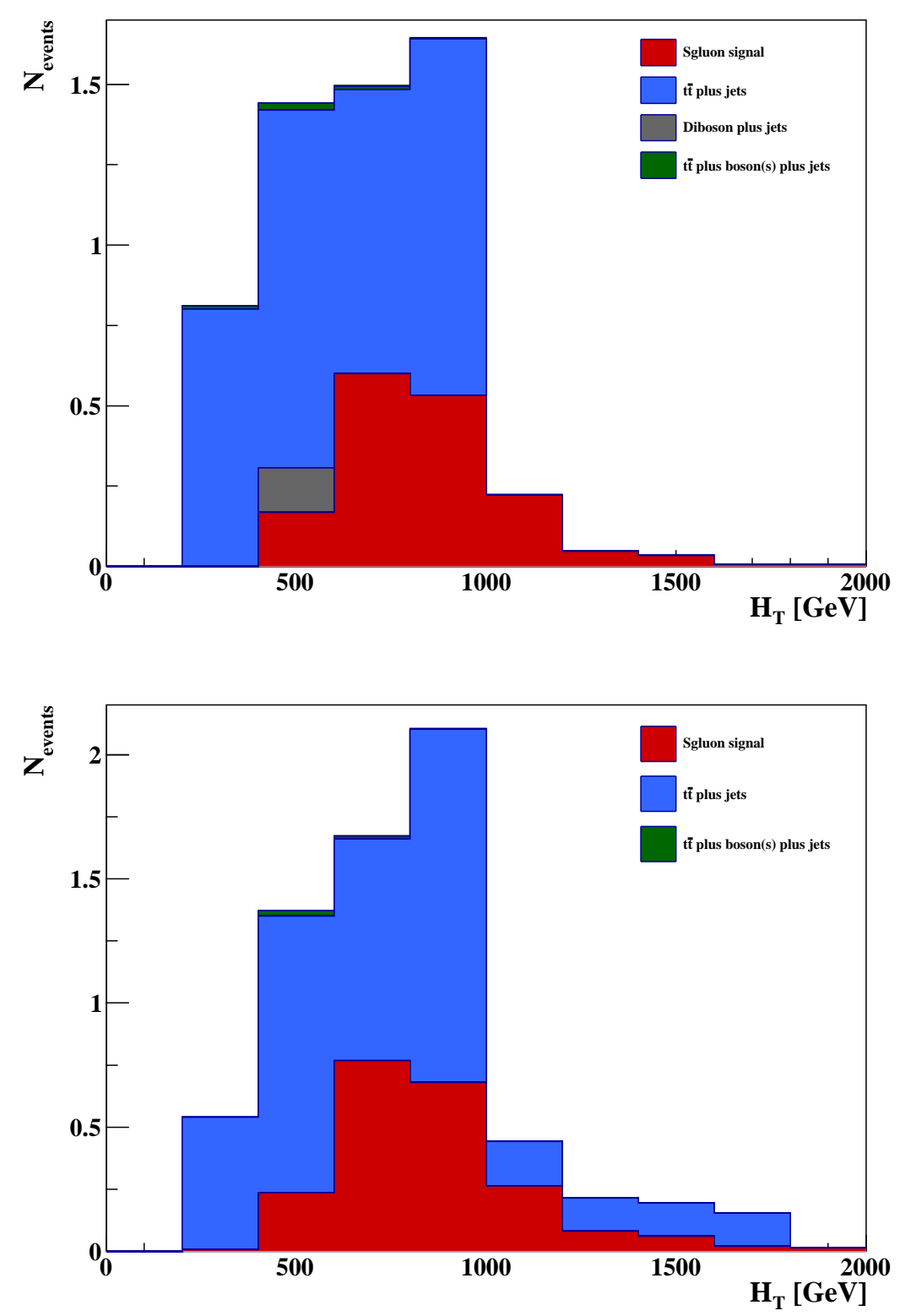

Fig. 5. Same as Figure 4 but with a weaker requirement of only six (seven) jets, three (two) of them being $b$-tagged jets in the upper (lower) panel of the figure. Events related to the production of a $t \bar{t}$ pair in association with one or several additional gauge boson are shown in green, whilst diboson events are shown in gray. 
Table 7. Number of events $N_{\text {events }}$ for both the different sources of background and for the signal after the whole set of selection cuts described in the text. Contributions of the single and diboson plus jets, the associated production of a $t \bar{t}$ pair and one or several gauge bosons,the single top plus jets and the QCD multijet channels are zero and thus not indicated. The results correspond to an integrated luminosity of $4 \mathrm{fb}^{-1}$ of proton-proton collisions at the LHC collider, running with a center-of-mass energy of $7 \mathrm{TeV}$.

\begin{tabular}{|c|c|}
\hline Event sample & $N_{\text {events after the selection cuts presented in the text }}$ \\
\hline \hline Top-antitop pairs plus jets & $0.5 \pm 0.3$ \\
\hline Total background & $0.5 \pm 0.3$ \\
Sgluon signal & $0.9 \pm 0.1$ \\
\hline
\end{tabular}

luminosity is not enough to obtain a sensitivity to the new physics signal. Therefore, only lighter sgluons would be accessible at $7 \mathrm{TeV}$. The results are however promising for the $2012 \mathrm{LHC}$ run at a center-of-mass energy of $8 \mathrm{TeV}$ and with an expected luminosity of $15 \mathrm{fb}^{-1}$.

\section{Conclusions}

In this work, we have illustrated how softly broken supersymmetric quantum field theories can be efficiently and easily implemented in the FEYNRULES package, exported then to Monte Carlo event generators and finally be ready for phenomenological investigations at hadron colliders.

Thanks to its superspace module, implementing a supersymmetric theory in FEYNRULES is reduced to the very simple task of providing the superfield and field content of the model, defining the free parameters appearing in the Lagrangian as well as providing the superpotential, in terms of superfields, and the soft supersymmetry breaking Lagrangian. The FEYNRULES program allows then to automatically expand the superfield Lagrangian in terms of the physical component fields, rendering it ready to be exported either to Monte Carlo event generators or to a PYTHON library dubbed the UFO model format, containing all the model information.

The UFO model format is agnostic of any assumption on the new physics model under consideration. Any interaction vertex, whatever is the number of incoming/outgoing particles, the included color and Lorentz structures, can be effectively exported, in contrast to the other model format used by automated Monte Carlo tools. The latter indeed rely on text files to be parsed and a set of hard-coded compliant color and Lorentz structures.

In this work, we have presented two phenomenological studies of collider signatures associated to interaction vertices with exotic color structures. Consequently, only the MADGRAPH 5 Monte Carlo generator, using the UFO format, is capable of generating events associated to such signatures. We therefore employ it to investigate monotop production in the framework of the minimal supersymmetric standard model with $R$-parity violation as well as multitop production in the context of the minimal $R$-symmetric supersymmetric model. We show that for the chosen bench- 
mark, the LHC is the perfect machine to unveil the presence of the corresponding new physics.

\section{Acknowledgements}

The author is extremely grateful to J. Andrea and E. Conte for their precious support in the phenomenological analyses performed in this work and to O. Mattelaer for his help with the generation of the events. The author also thanks the whole FeynRules, UFO and MadGraph 5 development teams, as well as J. Alwall, C. Duhr, F. Maltoni, K. Mawatari and M. Rausch de Traubenberg for discussions about the present manuscript. This work was supported by the Theory-LHC France Initiative of the CNRS/IN2P3.

\section{References}

1. H. P. Nilles, Phys. Rept. 110, 1 (1984).

2. H. E. Haber and G. L. Kane, Phys. Rept. 117, 75 (1985).

3. https://twiki.cern.ch/twiki/bin/view/AtlasPublic/SupersymmetryPublicResults

4. https://twiki.cern.ch/twiki/bin/view/CMSPublic/PhysicsResultsSUS

5. M. L. Mangano, M. Moretti, F. Piccinini, R. Pittau and A. D. Polosa, JHEP 0307, 001 (2003).

6. T. Gleisberg and S. Hoeche, JHEP 0812, 039 (2008).

7. A. Pukhov et al., arXiv:hep-ph/9908288.

8. E. Boos et al. [CompHEP Collaboration], Nucl. Instrum. Meth. A 534, 250 (2004).

9. A. Pukhov, arXiv:hep-ph/0412191.

10. A. Cafarella, C. G. Papadopoulos and M. Worek, Comput. Phys. Commun. 180, 1941 (2009).

11. T. Stelzer and W. F. Long, Comput. Phys. Commun. 81, 357 (1994).

12. F. Maltoni and T. Stelzer, JHEP 0302, 027 (2003).

13. J. Alwall et al., JHEP 0709, 028 (2007).

14. J. Alwall, P. Artoisenet, S. de Visscher, C. Duhr, R. Frederix, M. Herquet and O. Mattelaer, AIP Conf. Proc. 1078, 84 (2009).

15. J. Alwall, M. Herquet, F. Maltoni, O. Mattelaer and T. Stelzer, JHEP 1106, 128 (2011).

16. T. Gleisberg, S. Hoeche, F. Krauss, A. Schalicke, S. Schumann and J. -C. Winter, JHEP 0402, 056 (2004).

17. T. Gleisberg, S. Hoche, F. Krauss, M. Schonherr, S. Schumann, F. Siegert and J. Winter, JHEP 0902, 007 (2009).

18. M. Moretti, T. Ohl and J. Reuter, In *2nd ECFA/DESY Study 1998-2001* 1981-2009.

19. W. Kilian, T. Ohl and J. Reuter, Eur. Phys. J. C 71, 1742 (2011).

20. A. Semenov, Comput. Phys. Commun. 115, 124 (1998).

21. A. Semenov, Comput. Phys. Commun. 180, 431 (2009).

22. N. D. Christensen and C. Duhr, Comput. Phys. Commun. 180, 1614 (2009).

23. N. D. Christensen, P. de Aquino, C. Degrande, C. Duhr, B. Fuks, M. Herquet, F. Maltoni and S. Schumann, Eur. Phys. J. C 71 (2011) 1541.

24. N. D. Christensen, C. Duhr, B. Fuks, J. Reuter and C. Speckner, arXiv:1010.3251 [hep-ph].

25. C. Duhr and B. Fuks, Comput. Phys. Commun. 182, 2404 (2011).

26. F. Staub, Comput. Phys. Commun. 181, 1077 (2010) 
27. F. Staub, Comput. Phys. Commun. 182, 808 (2011).

28. A. Salam and J. A. Strathdee, Nucl. Phys. B 76, 477 (1974).

29. S. Ferrara, J. Wess and B. Zumino, Phys. Lett. B 51, 239 (1974).

30. T. Hahn and M. Perez-Victoria, Comput. Phys. Commun. 118, 153 (1999).

31. T. Hahn, Comput. Phys. Commun. 140 (2001) 418.

32. C. Degrande, C. Duhr, B. Fuks, D. Grellscheid, O. Mattelaer and T. Reiter, arXiv:1108.2040 [hep-ph].

33. G. Cullen, N. Greiner, G. Heinrich, G. Luisoni, P. Mastrolia, G. Ossola, T. Reiter and F. Tramontano, arXiv:1111.2034 [hep-ph].

34. P. de Aquino, W. Link, F. Maltoni, O. Mattelaer and T. Stelzer, arXiv:1108.2041 [hep-ph].

35. H. Murayama, I. Watanabe and K. Hagiwara, KEK-91-11.

36. K. Hagiwara, J. Kanzaki, Q. Li and K. Mawatari, Eur. Phys. J. C 56, 435 (2008).

37. K. Hagiwara, K. Mawatari and Y. Takaesu, Eur. Phys. J. C 71, 1529 (2011).

38. K. Mawatari and Y. Takaesu, Eur. Phys. J. C 71, 1640 (2011).

39. R. Barbier, C. Berat, M. Besancon, M. Chemtob, A. Deandrea, E. Dudas, P. Fayet and S. Lavignac et al., Phys. Rept. 420, 1 (2005).

40. P. Fayet, Nucl. Phys. B 113, 135 (1976).

41. L. Alvarez-Gaume and S. F. Hassan, Fortsch. Phys. 45, 159 (1997).

42. S. Y. Choi, M. Drees, A. Freitas and P. M. Zerwas, Phys. Rev. D 78, 095007 (2008).

43. S. Y. Choi, M. Drees, J. Kalinowski, J. M. Kim, E. Popenda and P. M. Zerwas, Phys. Lett. B 672, 246 (2009).

44. S. Y. Choi, M. Drees, J. Kalinowski, J. M. Kim, E. Popenda and P. M. Zerwas, Acta Phys. Polon. B 40, 1947 (2009).

45. S. Y. Choi, D. Choudhury, A. Freitas, J. Kalinowski, J. M. Kim and P. M. Zerwas, JHEP 1008, 025 (2010).

46. S. Schumann, A. Renaud and D. Zerwas, JHEP 1109, 074 (2011).

47. A. Salam and J. A. Strathdee, Nucl. Phys. B 87, 85 (1975).

48. P. Fayet, Nucl. Phys. B 90, 104 (1975).

49. G. D. Kribs, E. Poppitz and N. Weiner, Phys. Rev. D 78, 055010 (2008).

50. S. Y. Choi, D. Choudhury, A. Freitas, J. Kalinowski and P. M. Zerwas, Phys. Lett. B 697, 215 (2011) [Erratum-ibid. B 698, 457 (2011)].

51. T. Plehn and T. M. P. Tait, J. Phys. G G 36, 075001 (2009).

52. A. Brignole, J. A. Casas, J. R. Espinosa and I. Navarro, Nucl. Phys. B 666, 105 (2003).

53. M. Carena, K. Kong, E. Ponton and J. Zurita, Phys. Rev. D 81, 015001 (2010).

54. I. Antoniadis, E. Dudas, D. M. Ghilencea and P. Tziveloglou, Nucl. Phys. B 831, 133 (2010).

55. B. Fuks and M. Rausch de Traubenberg, Supersymétrie : exercices avec solutions (Ed. Ellipses, 2011).

56. E. Cremmer, B. Julia, J. Scherk, S. Ferrara, L. Girardello and P. van Nieuwenhuizen, Nucl. Phys. B 147, 105 (1979).

57. B. Zumino, Phys. Lett. B 87, 203 (1979).

58. E. Cremmer, S. Ferrara, L. Girardello and A. Van Proeyen, Phys. Lett. B 116, 231 (1982).

59. E. Cremmer, S. Ferrara, L. Girardello and A. Van Proeyen, Nucl. Phys. B 212, 413 (1983).

60. G. 't Hooft, Phys. Rev. Lett. 37, 8 (1976).

61. S. Dimopoulos and L. J. Hall, Phys. Lett. B 207, 210 (1988).

62. V. D. Barger, G. F. Giudice and T. Han, Phys. Rev. D 40, 2987 (1989). 
63. K. A. Intriligator, N. Seiberg and D. Shih, JHEP 0604, 021 (2006).

64. K. A. Intriligator, N. Seiberg and D. Shih, JHEP 0707, 017 (2007).

65. J. Polchinski and L. Susskind, Phys. Rev. D 26, 3661 (1982).

66. M. Dine and D. MacIntire, .Phys. Rev. D 46, 2594 (1992).

67. P. J. Fox, A. E. Nelson and N. Weiner, JHEP 0208, 035 (2002).

68. G. Corcella, I. G. Knowles, G. Marchesini, S. Moretti, K. Odagiri, P. Richardson, M. H. Seymour and B. R. Webber, JHEP 0101, 010 (2001).

69. M. Bahr, S. Gieseke, M. A. Gigg, D. Grellscheid, K. Hamilton, O. Latunde-Dada, S. Platzer and P. Richardson et al., Eur. Phys. J. C 58, 639 (2008).

70. T. Sjostrand, S. Mrenna and P. Z. Skands, JHEP 0605, 026 (2006).

71. T. Sjostrand, S. Mrenna and P. Z. Skands, Comput. Phys. Commun. 178, 852 (2008).

72. S. S. AbdusSalam, B. C. Allanach, H. K. Dreiner, J. Ellis, U. Ellwanger, J. Gunion, S. Heinemeyer and M. Kraemer et al., Eur. Phys. J. C 71, 1835 (2011).

73. M. Lancaster [Tevatron Electroweak Working Group and for the CDF and D0 Collaborations], arXiv:1107.5255 [hep-ex].

74. K. Nakamura et al. [Particle Data Group Collaboration], J. Phys. G G 37, 075021 (2010).

75. R. Barbieri and A. Masiero, Nucl. Phys. B 267, 679 (1986).

76. S. A. Abel, Phys. Lett. B 410, 173 (1997).

77. P. Slavich, Nucl. Phys. B 595, 33 (2001).

78. D. Chakraverty and D. Choudhury, Phys. Rev. D 63, 112002 (2001).

79. S. Bar-Shalom, G. Eilam and Y. D. Yang, Phys. Rev. D 67, 014007 (2003).

80. F. Zwirner, Phys. Lett. B 132, 103 (1983).

81. S. Dimopoulos and L. J. Hall, Phys. Lett. B 196, 135 (1987).

82. I. Hinchliffe and T. Kaeding, Phys. Rev. D 47, 279 (1993).

83. D. Chang and W. Y. Keung, Phys. Lett. B 389, 294 (1996).

84. K. Choi, K. Hwang and J. S. Lee, Phys. Lett. B 428 129, (1998).

85. G. Bhattacharyya and P. B. Pal, Phys. Lett. B 439, 81 (1998).

86. E. A. Baltz and P. Gondolo, Phys. Rev. D 57, 7601 (1998).

87. W. Porod, Comput. Phys. Commun. 153, 275 (2003).

88. W. Porod and F. Staub, arXiv:1104.1573 [hep-ph].

89. J. Pumplin, D. R. Stump, J. Huston, H. L. Lai, P. M. Nadolsky and W. K. Tung, JHEP 0207, 012 (2002).

90. B. Allanach et al. [R parity Working Group Collaboration], hep-ph/9906224

91. J. Andrea, B. Fuks and F. Maltoni, Phys. Rev. D 84, 074025 (2011).

92. S. Ovyn, X. Rouby and V. Lemaitre, arXiv:0903.2225 [hep-ph].

93. E. Conte, B. Fuks and G. Serret, in preparation.

94. E. L. Berger, B. W. Harris and Z. Sullivan, Phys. Rev. Lett. 83, 4472 (1999).

95. E. L. Berger, B. W. Harris and Z. Sullivan, Phys. Rev. D 63, 115001 (2001).

96. G. Aad et al. [Atlas Collaboration], Phys. Lett. B 701, 186 (2011).

97. S. Chatrchyan et al. [CMS Collaboration], JHEP 1108, 155 (2011).

98. CMS Collaboration, CMS-BTV-11-001.

99. CMS Collaboration, CMS-BTV-09-001. 